\title{
On the Resonance and Shelf/Open-Ocean Coupling of the Global Diurnal Tides
}

\author{
AARON W. SKIBA* \\ Department of Aerospace Engineering, University of Michigan, Ann Arbor, Michigan \\ LIBO ZENG* \\ Department of Physics, University of Michigan, Ann Arbor, Michigan
}

BRIAN K. ARBIC

Department of Earth and Environmental Sciences, University of Michigan, Ann Arbor, Michigan

MALTE MÜLlER ${ }^{+}$

School of Earth and Ocean Sciences, University of Victoria, Victoria, British Columbia, Canada

WILLIAM J. GODWIN ${ }^{\#}$

Department of Physics and Center for Ocean-Atmospheric Prediction Studies, The Florida State University, Tallahassee, Florida

(Manuscript received 17 March 2012, in final form 24 November 2012)

\begin{abstract}
The resonance of diurnal tidal elevations is investigated with a forward ocean tide model run in a realistic near-global domain and a synthesis of free oscillations (normal modes) computed for realistic global ocean geometries and ocean physics. As a prelude to performing the forward ocean tide simulations, the topographic wave drag, which is now commonly employed in forward ocean tide models, is tuned specifically for diurnal tides. The synthesis of global free oscillations predicts reasonably well the forward ocean diurnal tide model sensitivity to changes in the frequency, zonal structure, and meridional structure of the astronomical diurnal tidal forcing. Three global free oscillations that are important for understanding diurnal tides as a superposition of forced-damped, resonant, free oscillations are identified. An admittance analysis of the frequency sweep experiments demonstrates that some coastal locations such as the Sea of Okhotsk are resonant to diurnal tidal forcing. As in earlier work done with semidiurnal tides, a series of simulations are performed in which regions possessing significant coastal diurnal tides are blocked out. The largest perturbations to the open-ocean diurnal tides take place in Blocked Sea of Okhotsk experiments. Lesser but still significant perturbations also arise from the blocking out of other regions of large diurnal tidal elevations or dissipation. Interpretation of the results is made more complex, however, by the fact that substantial perturbations also arise from blocking out regions where neither tidal elevations nor dissipation are large. The "blocking" experiments are relevant to understanding tides of the ice age, during which lower sea levels entail a reduced area of continental shelves.
\end{abstract}

* These authors contributed equally to this work.

+ Current affiliation: Research and Development Department, Norwegian Meteorological Institute, Oslo, Norway.

\# Current affiliation: Department of Nuclear and Radiological Engineering, University of Florida, Gainesville, Florida.

Corresponding author address: Dr. Brian K. Arbic, Department of Earth and Environmental Sciences, University of Michigan, Ann Arbor, MI 48109-1005.

E-mail: arbic@umich.edu

\section{Introduction}

This paper examines the resonance of diurnal tides in the open and coastal oceans and the coupling between the open-ocean and coastal diurnal tides. We are motivated by the inherent interest in understanding the global tidal system and by recent studies which strongly suggest that tides of the ice age, during which lower sea levels implied a much reduced area of continental shelves, were much larger than those of 
today (e.g., Thomas and Sündermann 1999; Egbert et al. 2004; Arbic et al. 2004b, 2008; Uehara et al. 2006; Griffiths and Peltier 2008, 2009; Green 2010; Hill et al. 2011).

The topic of tidal resonance has a long and rich history. The open-ocean tides have been argued to be near resonant in both admittance studies of observations (Wunsch 1972; Garrett and Greenberg 1977; Heath 1981) and in studies noting the similarities between free oscillations computed in both idealized and realistic geometries (Rao 1966; Longuet-Higgins and Pond 1970; Platzman et al. 1981; Platzman 1991; Zahel and Müller 2005; Müller 2007) and the actual ocean tides. A number of studies have argued that selected coastal regions around the globe are resonant to semidiurnal or diurnal tidal forcing (e.g., Garrett 1972; Clarke 1991; Sutherland et al. 2005; Arbic et al. 2007; Cummins et al. 2010, among others).

Here, as in two previous papers, we add to the discussion of tidal resonance through experimentation with global forward tide models. Arbic et al. (2009, hereafter AKG) and Arbic and Garrett (2010, hereafter AG) explored the resonant attributes of the global semidiurnal tides. Following up on our work in AKG and AG, here we conduct a systematic exploration of resonance in a forward near-global model of the diurnal tides. The forward ocean tide model simulations are interpreted primarily with a model of free oscillations computed for realistic global ocean geometries and ocean physics and including frictional terms and the full self-attraction and loading effect (Müller 2007). Note that the set of free oscillations are often called "normal modes", and we will at times refer to the global free oscillations model as a "modal synthesis" model. Note also that both the forward ocean tide model and the set of free oscillations are "numerical." To avoid confusion, in this paper the term "numerical" will not generally be used in the description of either model.

Ocean tides are impacted by several factors. For instance, they are influenced by solid-earth body tides and the self-attraction and loading (SAL) term (Hendershott 1972), as well as by damping (quadratic bottom boundary layer drag and topographic internal wave drag). These factors will be described later in the paper. Below we describe the impact of oceanic length and depth scales.

Ocean depths and length scales exert a critical influence on ocean tides. The water column depth $H$ and gravitational acceleration $g$ set the tidal phase speed $\sqrt{g H}$. A nonrotating basin of length $L$ that is closed at both ends experiences the classical half-wavelength resonance when

$$
\frac{\omega L}{\sqrt{g H}}=n \pi
$$

where $n$ is an integer and $\omega$ is the tidal forcing frequency (e.g., Proudman 1953; Defant 1961; Godin 1988). A simple model that has been used to explain regions of large resonant coastal tides consists of a small nonrotating basin with one end closed (the continent) and the other end open to, and forced by, the open ocean. The classical quarter-wavelength resonance arises in the latter case when

$$
\frac{\omega L}{\sqrt{g H}}=(2 n-1) \frac{\pi}{2}
$$

(e.g., Proudman 1953; Defant 1961; Godin 1988). All of this implies that $g$, the forcing frequency $\omega$, water depth $H$, and basin (or coastal) scale $L$ are critical controls on tides. A more complete description of tidal resonance must account for the effects of rotation as well as the values of $g, \omega, H$, and $L$. Gravity waves propagating along boundaries in a rotating basin take the form of Kelvin waves, which are highly prominent in maps and animations of both semidiurnal and diurnal tides. In the presence of rotation, the resonant normal modes of an idealized basin are quite different from those in a nonrotating basin (Rao 1966). Of course, the normal modes of the ocean computed under realistic geometries, and utilized in this paper, account for the Coriolis force due to the earth's rotation.

The structure of the tides is also dependent upon the spatial structure in the astronomical tidal forcing. The equilibrium tidal forcing of the largest semidiurnal tides in the ocean $\left(M_{2}, S_{2}, N_{2}\right.$, and $\left.K_{2}\right)$ is proportional to the spherical harmonic $Y_{2}^{2}(\phi, \lambda)$, where $\phi$ is latitude and $\lambda$ is longitude (Laplace 1775, 1776; Cartwright 1977; Hendershott 1981; Arfken and Weber 2001). The equilibrium tidal forcing of the largest diurnal tides in the ocean $\left(K_{1}, O_{1}, P_{1}\right.$, and $\left.Q_{1}\right)$ is proportional to the spherical harmonic $Y_{2}^{1}(\phi, \lambda)$ (Laplace 1775, 1776; Cartwright 1977; Hendershott 1981; Arfken and Weber 2001). Resonant free oscillations of the ocean have characteristic spatial structures and frequencies (Platzman 1991; Müller 2009) whose alignment with the spatial structure and frequency of the astronomical tidal forcing determines the oceanic response to tidal forcing.

The factors that control diurnal tides will be examined in detail here. In the forward ocean tide model we vary the frequency $\omega$, zonal spatial structure, and meridional spatial structure of the astronomical diurnal tidal forcing. The set of global free oscillations offers predictions of tidal sensitivity to forcing frequency. The spatial structures associated with the global free oscillations 
also permit an examination of the sensitivities of diurnal tides to both zonal and meridional spatial structure in the astronomical tidal forcing.

Because tides strongly depend upon water depths and basin length scales, they respond sensitively to changes in coastal ocean geometry. An additional factor in the sensitivity of open-ocean tides to changes in coastal regions is the substantial fraction of globally integrated tidal dissipation taking place in such regions (Egbert and Ray 2003). As previously noted, forward tide models suggest that tides of the ice ages were much larger than those of today. During ice ages, lower sea levels led to the removal of significant areas of present-day shelves. Motivated by this reduction in shelf area, and by the inherent interest in understanding the coupling of openocean and shelf tides, AKG and AG examined the response of open-ocean semidiurnal tides to the blocking out of regions of significant coastal semidiurnal tides. Here we perform experiments in which regions of significant coastal diurnal tides are blocked out.

It can be difficult to ascertain whether changes in geometry or in dissipation lie behind the impacts of the blocking experiments. The blocking experiments for semidiurnal tides in $\mathrm{AKG}$ and $\mathrm{AG}$ were interpreted with two highly simplified analytical models of open ocean-coastal tidal coupling. The coupled oscillator model of AG considers the shelf and the open ocean to each be a damped single spring system; the two are then coupled together. The one-dimensional nonrotating shallow water model of AKG considers the open ocean and shelf to both be boxes. Their dynamics are coupled because the smaller box is open to the larger box on one side. In both of these analytical models, if the natural periods of the shelf and open ocean are both set to be near the forcing period, removal of the shelf leads to substantial perturbations-generally, increases-in the open-ocean tides. This suggests that even a very small oscillator can have a profound effect on a nearby much larger oscillator it is coupled to, especially if both are near resonance. The AG and AKG analytical models allow for both the effects of coastal resonance and coastal tidal dissipation on the open-ocean tides; see for instance Eqs. (12)-(18) of AG.

In this paper we will show that the Sea of Okhotsk is resonant to diurnal tidal forcing and that removal of the Sea of Okhotsk leads to larger perturbations to the open-ocean diurnal tides than does removal of other coastal regions of similar area. This result is consistent with the simple interpretive models of AKG and AG, which continue to predict a large "back effect" of resonant coastal tides upon the open ocean when their governing parameters are changed from those suitable for semidiurnal tides to those suitable for diurnal tides.
However, we will not utilize the simple interpretive models in this paper nearly as extensively as in AKG and $\mathrm{AG}$, in part for the sake of brevity, in part owing to a reviewer who objected to the lack of rotation effects in either simple model, and in part due to the fact that even removal of regions of small coastal diurnal tidal elevations and dissipation can significantly impact the openocean tides (a prediction not consistent with the simple models). Note that recomputing the normal modes of Müller (2009) under various blocking scenarios or other altered ocean geometries would be far too time consuming to be practical for the present paper.

This paper is organized as follows. In section 2, we discuss the modal synthesis (global free oscillation) model. Details of the forward ocean tide model, the tuning required to optimize its topographic internal wave drag for diurnal tides, and its accuracy with respect to satellite-altimetry-constrained tide models, are presented in section 3. Section 4 presents the sensitivity tests to the frequencies and spatial structures in the tidal forcing, conducted with the forward ocean tide model and the set of global free oscillations. Section 4 also displays the results of the blocking experiments. Finally, section 4 demonstrates that the Sea of Okhotsk is resonant to diurnal tidal forcing and that blocking out the Sea of Okhotsk perturbs the open-ocean tides more than blocking out other regions of similar area. In section 5 we summarize our results.

\section{The modal synthesis model}

Ocean tides have been described (e.g., Platzman 1991) as a superposition of global free oscillations. Here we use a set of barotropic global free oscillations and their adjoint counterparts computed on a $1^{\circ}$ horizontal grid including the North Pole, linearized friction terms, and full self-attraction and loading effects (Müller 2007).

In the synthesis procedure we write the $k$ th free oscillation as a vector

$$
\mathbf{x}_{k}=\left[\zeta_{k}(\phi, \lambda), u_{k}(\phi, \lambda), v_{k}(\phi, \lambda)\right],
$$

where $\phi$ is latitude, $\lambda$ is longitude, $\zeta_{k}$ is the sea surface elevation, $u_{k}$ is the zonal velocity, and $v_{k}$ is the meridional velocity. The free oscillations are the solutions of the homogeneous tidal equations, which are written in general operator notation equation as

$\frac{\partial}{\partial t} \mathbf{x}_{k} e^{-i \omega_{k} t}+\mathcal{L} \mathbf{x}_{k} e^{-i \omega_{k} t}=0 \rightarrow\left(\mathcal{L}-i \omega_{k}\right) \mathbf{x}_{k}=0$,

where the operator $\mathcal{L}$ represents the linear shallow water dynamics with rotation effects included and $\omega_{k}$ represents 
the $k$ th complex eigenvalue, $i \omega_{k}=\omega_{k, 1}+i \omega_{k, 2}$, with the oscillatory part $\omega_{k, 2}$ and the damping part $\omega_{k, 1}$.

The ocean tides are the solutions of the inhomogeneous tidal equations; that is,

$$
\begin{aligned}
\frac{\partial}{\partial t} \mathbf{x}_{\text {tide }} e^{-i \omega t}+\mathcal{L} \mathbf{x}_{\text {tide }} e^{-i \omega t} & =\mathbf{F}_{\text {tide }} e^{-i \omega t} \\
\rightarrow(\mathcal{L}-i \omega) \mathbf{x}_{\text {tide }} & =\mathbf{F}_{\text {tide }}
\end{aligned}
$$

where $\mathbf{F}_{\text {tide }} e^{-i \omega t}$ represents astronomical tidal forcing of frequency $\omega$. Generally, every tidal solution $\mathbf{x}_{\text {tide }}$ can be expressed by a superposition of free oscillations $\mathbf{x}_{k}$ :

$$
\mathbf{x}_{\text {tide }}=\sum_{k=1}^{\infty} a_{k} \mathbf{x}_{k},
$$

where $a_{k}$ are the complex weighting coefficients. A direct computation of the weighting coefficients is only possible with the knowledge of the eigenvectors $\hat{\mathbf{x}}_{k}$ of the adjoint eigenproblem

$$
\left(\overline{\mathcal{L}-i \omega_{k}}\right) \hat{\mathbf{x}}_{k}=0 .
$$

If dissipative terms are neglected in the tidal dynamics the adjoint eigenvectors $\hat{\mathbf{x}}_{k}$ are simply the complex conjugates of their corresponding eigenvectors. This relationship was utilized by Platzman (1984) for a tidal synthesis procedure. However, in the present study dissipative terms are considered, and thus the operator $\mathcal{L}$ becomes nonself-adjoint and the adjoint eigensolutions have to be determined separately. These pairs of eigensolutions are, when properly normalized, biorthonormal $\left(\left\langle\mathbf{x}_{i}, \hat{\mathbf{x}}_{j}\right\rangle=0\right.$ when $\left.i \neq j\right)$, where the angle brackets represent an arbitrary scalar product. Combining Eqs. (4), (5), (6), and (7) yields

$$
a_{k}=\frac{1}{i\left(\omega_{k}-\omega\right)} \cdot\left\langle\mathbf{F}_{\text {tide }}, \hat{\mathbf{x}}_{k}\right\rangle .
$$

In the following we use the biorthonormal systems of eigenfunctions described by Müller (2008, 2009). We refer to these publications for a more detailed discussion of the adjoint solutions and the definition of the scalar product.

As described in Eq. (8), the sensitivity of the ocean's response to the tidal forcing is determined by the distance between the forcing and free oscillation frequency $\left|\omega-\omega_{k, 2}\right|$, the free oscillation decay time $1 /\left(2 \omega_{k, 1}\right)$ and the shape factor $\left\langle\mathbf{F}_{\text {tide }}, \hat{\mathbf{x}}_{k}\right\rangle$ of the free oscillation. The latter describes the spatial coherence of the tidal forcing and the adjoint free oscillation. With the knowledge of the free oscillations $\left\{\mathbf{x}_{k}\right\}_{k=1, \infty}$ and their adjoint counterparts $\left\{\hat{x}_{k}\right\}_{k=1, \infty}$ every forced barotropic oscillation can be obtained by superposition via Eq. (6). However, a subset of oscillations in the period range from 10 to $80 \mathrm{~h}$ is sufficient for synthesis of the diurnal and semidiurnal tides. Experiments in which we vary the forcing frequency $\omega$ and the zonal and meridional wavelengths of the forcing $\mathbf{F}_{\text {tide }}$ are discussed in sections $4 \mathrm{a}, 4 \mathrm{~b}$, and $4 \mathrm{c}$, respectively.

\section{Forward ocean tide model}

This section discusses the forward ocean tide model. As in AKG and AG, the forward model is the tide model of Arbic et al. (2004a, hereafter AGHS). A few details of the model are given here; AGHS can be consulted for full descriptions of model details including the preparation of topography. In this paper we use a latitudelongitude grid running from $86^{\circ} \mathrm{S}$ to $82^{\circ} \mathrm{N}$, as in AGHS, $\mathrm{AKG}$, and $\mathrm{AG}$. In contrast to most of the simulations in AGHS, AKG, and AG, the simulations here use $1 / 8^{\circ}$ horizontal resolution instead of $1 / 2^{\circ}$ resolution. Consistent with Egbert et al. (2004), in Arbic et al. (2008) and Müller et al. (2011) we found that this reduced the error of the forward model measured with respect to altimetryconstrained models. To allow for spinup, all of the simulations in this paper are run for at least 60 days, and analysis is undertaken on the latter part of the record (last 1-4 days). Note that, since all of the experiments in this paper are forced by just one frequency, there are no difficulties in separating nearby frequencies as there are in the actual ocean.

The governing equations for the forward ocean tide model are given in AGHS. The astronomical diurnal equilibrium tidal forcing $\eta_{\mathrm{EQ}}$ is

$$
\eta_{\mathrm{EQ}}=\left(1+k_{2}-h_{2}\right) A_{\text {astronomical }} \sin (2 \phi) \cos (\omega t+\lambda),
$$

where $k_{2}$ and $h_{2}$ are Love numbers accounting for effects of the solid-earth body tide (Hendershott 1972), $A_{\text {astronomical }}$ is the astronomical forcing amplitude, $\phi$ is latitude, $\omega$ is the forcing frequency, $t$ is time, and $\lambda$ is longitude. Except where noted, we set $A_{\text {astronomical }}$, $1+k_{2}-h_{2}$, and $\omega$ to the $K_{1}$ values of $14.1565 \mathrm{~cm}, 0.736$, and $0.7292117 \times 10^{-4} \mathrm{~s}^{-1}$, respectively, in all of the forward diurnal tide model simulations in this paper.

Our forward ocean tide model incorporates a parameterized topographic internal wave drag scheme (Garner 2005), which is motivated by inferences from both in situ microstructure data (Polzin et al. 1997) and satellite-altimetry-constrained tide models (Egbert and Ray 2000,2001,2003) of enhanced dissipation in regions 
of rough topography. Parameterized topographic internal wave drag improves the accuracy of forward tide models (Jayne and St. Laurent 2001; Carrere and Lyard 2003; Egbert et al. 2004; AGHS; Lyard et al. 2006; Uehara et al. 2006; Griffiths and Peltier 2008, 2009; Green 2010) because tidal amplitudes are controlled by the strength of the topographic drag (Egbert et al. 2004; AGHS). In AGHS the wave drag was tuned to minimize the discrepancy in open-ocean $M_{2}$ elevations between the forward model and the satellite-altimetry-constrained GOT99 model of Ray (1999). As discussed below, in the present paper we retain the same topographic internal wave drag scheme, but optimized for $K_{1}$ tides. Simulations for the present paper are conducted with a cutoff depth for activating topographic internal wave drag of $100 \mathrm{~m}$, rather than $1000 \mathrm{~m}$ as in AGHS. As noted in Arbic et al. (2008) this improves the accuracy of the forward model and allows for a smaller value of the multiplicative factor discussed in AGHS. For all of the diurnal simulations presented in this paper, the multiplicative factor is set to 3 . It should be noted that, despite the different tuning factors in the AGHS scheme and say, for instance, the wave drag scheme in Jayne and St. Laurent (2001), the spatially averaged drag strengths of the two schemes are very similar, as pointed out by AGHS. Thus, the suggestion is that a certain average strength of drag is needed to dissipate the proper amount of energy, regardless of the exact details of the wave drag scheme used.

The effects of deformation of the solid earth by the loading of ocean tides and the perturbations in the gravitational potential due to the self-gravitation of both ocean tides and the load-deformed solid earth are collectively known as the self-attraction and loading (SAL) term (Hendershott 1972). As in AGHS (see also Egbert et al. 2004), the SAL term is computed here with an iterative method. As in AGHS and AKG, we display results from the third iteration of the SAL term.

\section{Tuning the forward model for $K_{1}$ tides}

This subsection discusses the tuning of the forward model's parameterized topographic internal wave drag scheme for $K_{1}$ tides. Our goal is to minimize discrepancies between the forward model and satellite-altimetryconstrained tide models. Here we examine kinetic energies as well as elevations. The $K_{1}$ kinetic energies in forward global tide models with drag schemes optimally tuned for $M_{2}$ elevations are weaker than $K_{1}$ kinetic energies found in either current meter observations or altimetry-constrained models (P. Timko 2011, personal communication; Timko et al. 2012). We conjecture that the low diurnal kinetic energies result from the fact that models employing a wave drag tuned for $M_{2}$ will damp $K_{1}$ too strongly. Free internal waves are not generated by diurnal tides at topography poleward of about $30^{\circ}$ latitude because of the requirement that the Coriolis parameter $f$ be less than the forcing frequency (Gill 1982). As noted by, for instance, Jayne and St. Laurent (2001), topographic internal wave drag should be corrected by a frequency-dependent factor $\sqrt{\omega^{2}-f^{2}} / \omega$. We therefore tested a solution in which the wave drag set up for $M_{2}$ and with a multiplicative factor of 3 is then multiplied by a correction factor $\sqrt{\omega_{K_{1}}^{2}-f^{2}} \omega_{M_{2}} / \sqrt{\omega_{M_{2}}^{2}-f^{2}} \omega_{K_{1}}$, where $\omega_{K_{1}}$ and $\omega_{M_{2}}$ are the forcing frequencies for $K_{1}$ and $M_{2}$, respectively. This correction factor is the ratio of the frequency-dependent factor for $K_{1}$ divided by the factor for $M_{2}$ - the latter is employed in the wave drag scheme tuned for $M_{2}$. This method is referred to in Table 1 as " $K_{1}$ correction method 1." We also examined changing the frequency-dependent factor to the $K_{1}$ value (" $K_{1}$ correction method 2" in Table 1 ), which is not exactly the same as correcting the full drag used in $M_{2}$ simulations by $\sqrt{\omega_{K_{1}}^{2}-f^{2}} \omega_{M_{2}} / \sqrt{\omega_{M_{2}}^{2}-f^{2}} \omega_{K_{1}}$ because the Garner (2005) drag scheme includes nonlinear as well as linear terms.

To compare the forward ocean tide model (and modal synthesis model) with satellite-altimetry-constrained tide models we compute several diagnostics. We measure the rms area-averaged magnitude of tidal elevations by the diagnostic

$$
\eta_{\mathrm{rms}}=\sqrt{\left[\iint \eta^{2} d A\right] / \iint d A},
$$

where $\eta$ is the sea surface elevation, square brackets denote time averaging over one tidal period, and $d A$ is an element of area. We measure the discrepancy between the forward or modal synthesis models and a benchmark altimetry-constrained model having elevations $\eta_{\text {BENCHMARK }}$ via

$$
\text { discrepancy }=\sqrt{\left[\iint\left(\eta-\eta_{\text {BENCHMARK }}\right)^{2} d A\right] / \iint d A} .
$$

As in AGHS, the percent variance captured is then computed by $100\left[1-\left(\text { discrepancy } / \eta_{\mathrm{rms}}\right)^{2}\right]$. As in AGHS and $\mathrm{AKG}$, the $\eta_{\mathrm{rms}}$, discrepancy, and percent variance captured values are computed over latitudes equatorward of $66^{\circ}$, the latitudes covered by the Ocean Topography Experiment (TOPEX)/Poseidon altimeter, and, unless otherwise noted, are computed only over locations where the water depths exceed $1000 \mathrm{~m}$ (i.e., shallow regions are excluded). The grid points over which water depths exceed $1000 \mathrm{~m}$ will vary slightly in 
TABLE 1. First column: List of diagnostics used to measure performance of forward model simulations of the $K_{1}$ tide. Second column: diagnostics computed from a forward $K_{1}$ simulation with "original drag," that is, a drag scheme optimally tuned for $M_{2}$ elevations. Third column: same as second column only with the $M_{2}$ drag scheme corrected by the factor $\sqrt{\omega_{K_{1}}^{2}-f^{2}} \omega_{M_{2}} / \sqrt{\omega_{M_{2}}^{2}-f^{2}} \omega_{K_{1}}$ discussed in section 3 . Fourth column: same as second column only with the value of $\omega$ in the frequency-dependent factor of the drag scheme changed to the value corresponding to the $K_{1}$ tide. Fifth column: diagnostics computed from TPXO (Egbert et al. 1994) output. Values for "Dissipation" and "Percent dissipation deep" were retrieved from Table 1 of Egbert and Ray (2003). Sixth column: same as fifth column only with data from GOT99 (Ray 1999 and Table 1 of Egbert and Ray 2003). "Percent variance captured" of the forward ocean tide model is computed with respect to GOT99 (Ray 1999) in waters deeper than $1000 \mathrm{~m}$ equatorward of $66^{\circ}$.

\begin{tabular}{|c|c|c|c|c|c|}
\hline Diagnostics & Original drag & $K_{1}$ correction method 1 & $K_{1}$ correction method 2 & TPXO & GOT99 \\
\hline$\eta_{\mathrm{rms}}(\mathrm{cm})$ & 9.44 & 9.51 & 9.42 & 9.46 & 9.54 \\
\hline Percent variance captured & 97.2 & 95.0 & 94.5 & - & - \\
\hline Kinetic energy $\left(10^{15} \mathrm{~J}\right)$ & 29.4 & 37.9 & 37.3 & 41.0 & - \\
\hline Percent kinetic energy shallow & 36.3 & 44.4 & 44.4 & 45.1 & - \\
\hline Potential energy $\left(10^{15} \mathrm{~J}\right)$ & 22.8 & 24.3 & 23.9 & 22.6 & 22.4 \\
\hline Power input (TW) & 0.334 & 0.313 & 0.316 & 0.340 & 0.347 \\
\hline Dissipation (TW) & 0.337 & 0.312 & 0.315 & 0.343 & - \\
\hline Percent dissipation deep & 26.1 & 16.8 & 19.2 & 11.3 & 16.9 \\
\hline
\end{tabular}

some of the blocking experiments, in which a small number of gridpoints deeper than $1000 \mathrm{~m}$ are blocked out. The $\eta_{\text {rms }}$ values computed from the forward ocean tide model simulations are compared against those from GOT99 in Table 1. We compute the area-integrated kinetic energy (KE) and available potential energy (APE) by

$$
\begin{aligned}
\mathrm{KE} & =\frac{1}{2} \rho_{0} \iint(H+\eta) \mathbf{u} \cdot \mathbf{u} d A \text { and } \\
\mathrm{APE} & =\frac{1}{2} \rho_{0} g \iint \eta^{2} d A,
\end{aligned}
$$

where $\rho_{0}=1035 \mathrm{~kg} \mathrm{~m}^{-3}$ is an average seawater density and $H$ is the resting water depth. The metric denoted by "Power input" is computed from

$$
P_{\text {input }}=\rho_{0} g \iint\left(\eta_{\mathrm{EQ}} \frac{\partial \eta}{\partial t}\right) d A,
$$

(Egbert and Ray 2001 and references therein), where $\eta_{\mathrm{EQ}}$ is the equilibrium tide. The metric denoted by "Dissipation" is computed from the dissipation summed over contributions due to the topographic wave drag, quadratic bottom boundary layer drag, and eddy viscosity, using code developed in Simmons et al. (2004).

A summary of these diagnostics computed from a $K_{1}$ simulation with the original wave drag (tuned for $M_{2}$, as in AGHS), $K_{1}$ simulations with $K_{1}$ correction methods 1 and 2 , and output from the altimetry-constrained models TPXO (Egbert et al. 1994) and GOT99 (Ray 1999) is provided in Table 1. Some of the TPXO and GOT99 values are taken from Table 1 in Egbert and Ray (2003). The quantities which can be computed strictly from elevations $-\eta_{\mathrm{rms}}$, percent variance captured, potential energy, and "Power input"-compare well to TPXO and GOT99 for all three forward simulations. "Dissipation" matches "Power input" well, as it must in energy balance. In the simulation utilizing wave drag tuned for $M_{2}$ ("Original drag"), the $K_{1}$ kinetic energy is, indeed, much weaker than in TPXO, as anticipated. The percent of kinetic energy in shallow waters is also much smaller than the value in TPXO. In addition, the percent of dissipation in deep waters is larger than in TPXO or GOT99. The two solutions with drag corrected for $K_{1}$ match the TPXO and GOT99 values more closely overall, although the "Percent variance captured," "Power input," and "Dissipation" diagnostics actually compare somewhat less well than in the solution with $M_{2}$ drag. The " $K_{1}$ correction method 1" matches the percentage of deep dissipation better than " $K_{1}$ correction method 2"; " $K_{1}$ correction method 1" is therefore taken as the baseline, or nominal, $K_{1}$ solution in the remainder of the paper.

The $K_{1}$ elevation amplitudes from GOT99 (Ray 1999), the optimally tuned forward ocean tide model (" $K_{1}$ correction method 1 "), and the modal synthesis model are displayed in Fig. 1. The three models match each other fairly well, although differences are clearly visible. Note, for instance, the overly strong tides in the southern Sea of Okhotsk in the forward ocean tide model. The $\eta_{\mathrm{rms}}$ and percent variance captured diagnostics for the modal synthesis model are given in Table 2.

The dissipation map from our optimally tuned forward model of $K_{1}$ is shown in Fig. 2. It looks qualitatively similar to the $K_{1}$ dissipation map in Egbert and Ray (2003), but there are notable differences in some locations, for example, Patagonia. Dissipation from quadratic bottom boundary layer drag is substantial in 
(a) GOT99

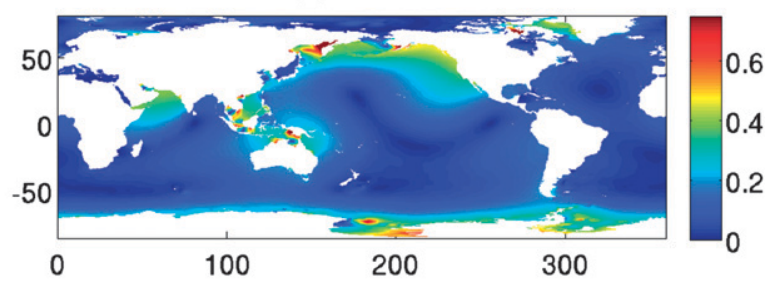

(b) Forward Model

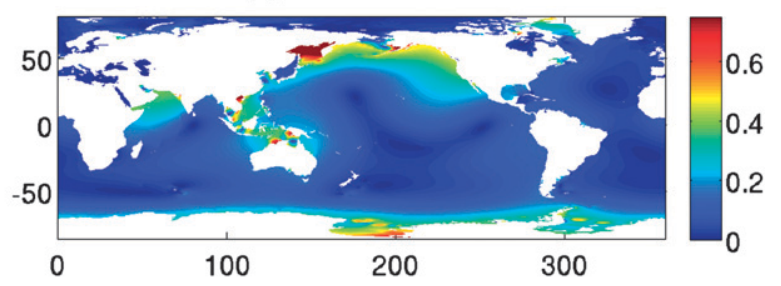

(c) Modal Synthesis Model

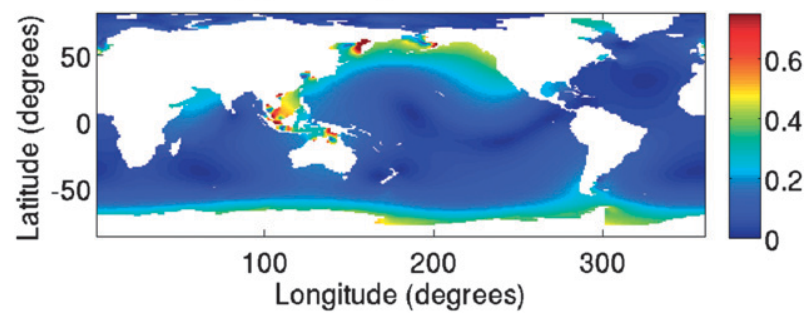

FIG. 1. Amplitude of $K_{1}$ elevation (m) in (a) GOT99 (Ray 1999), a highly accurate satellite-altimetry-constrained tide model, (b) optimally tuned forward ocean tide simulation $\left(K_{1}\right.$ correction method 1), referred to as the "nominal" simulation in the text, and (c) the modal synthesis (global free oscillation) model.

the Sea of Okhotsk and other shallow sea regions. The topographic internal wave drag dissipates substantial energy in the open Pacific and Indian Oceans in latitudes equatorward of $30^{\circ}$.

\section{Results of sensitivity experiments}

In this section, we discuss the sensitivity of the forward ocean tide model and the global free oscillation model to changes in the frequency, zonal structure, and meridional structure in the astronomical diurnal tidal forcing. Our initial emphasis is on the open ocean, but later in the section we present an analysis of the frequency sweep in the Sea of Okhotsk, which is shown to be resonant to diurnal tidal forcing. We conclude this section by examining the sensitivity of open ocean diurnal tides to the removal of regions of strong coastal diurnal tides such as the Sea of Okhotsk.

\section{a. Sensitivity to forcing frequency}

This subsection focuses on simulations of the forward and modal synthesis models with the same basin
TABLE 2. Second column: diagnostics computed from the modal synthesis model's simulation of the $K_{1}$ tide. Third column: diagnostics computed from the satellite-altimetry-constrained tide model GOT99 (Ray 1999) after interpolation to a $1^{\circ}$ grid.

\begin{tabular}{lcc}
\hline \hline \multicolumn{1}{c}{ Diagnostics } & Modal synthesis model & GOT99 \\
\hline$\eta_{\text {rms }}(\mathrm{cm})$ & 9.36 & 9.55 \\
Percent variance captured & 61.8 & - \\
\hline
\end{tabular}

geometry and topography as the nominal solution, but in which the forcing frequency $\omega$ in the astronomical diurnal tidal forcing Eq. (9) is allowed to vary. For the sake of brevity, global amplitude maps of these simulations are not shown. Instead, in Fig. 3a, we plot global $\eta_{\text {rms }}$ values from the forward ocean tide model and modal synthesis model (averaged over latitudes equatorward of $66^{\circ}$ and in water depths exceeding $1000 \mathrm{~m}$ ), as a function of the forcing period. Both the forward and modal synthesis models display clear peaks near 22 and $33 \mathrm{~h}$, and a less prominent peak near $26 \mathrm{~h}$. To assess the realism of the frequency sweeps conducted with the forward model, Fig. 3a includes results on the diurnal constituents $Q_{1}, O_{1}$, $P_{1}$, and $K_{1}$ from the satellite-altimetry-constrained tide models TPXO7.2 (Egbert et al. 1994) and GOT99 (Ray 1999). The TPXO7.2 and GOT99 results have been rescaled by the ratio of $A\left(1+k_{2}-h_{2}\right)$ evaluated for $K_{1}$ to $A\left(1+k_{2}-h_{2}\right)$ evaluated for the constituent in question (see Table 3), the underlying assumption being that tides are linear to first order. Discrepancies between the forward ocean tide model and the altimetry-constrained models (and between the two altimetry-constrained models themselves) are clearly seen. The agreement between the forward and modal synthesis models and the altimetry-constrained models is close for $K_{1}$ and

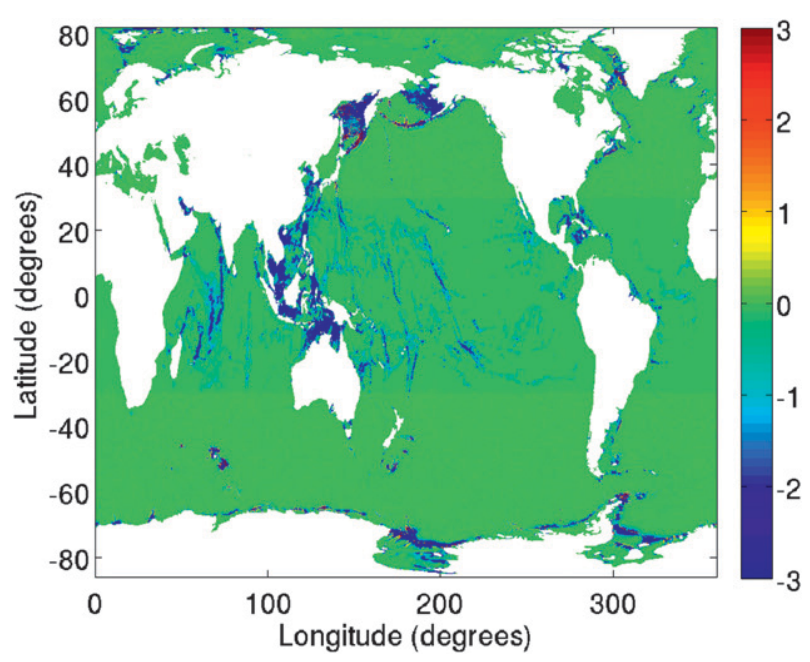

FIG. 2. Dissipation map (milliwatts per squared meter) of $K_{1}$ from our "nominal" simulation. 
(a) Globally averaged amplitude

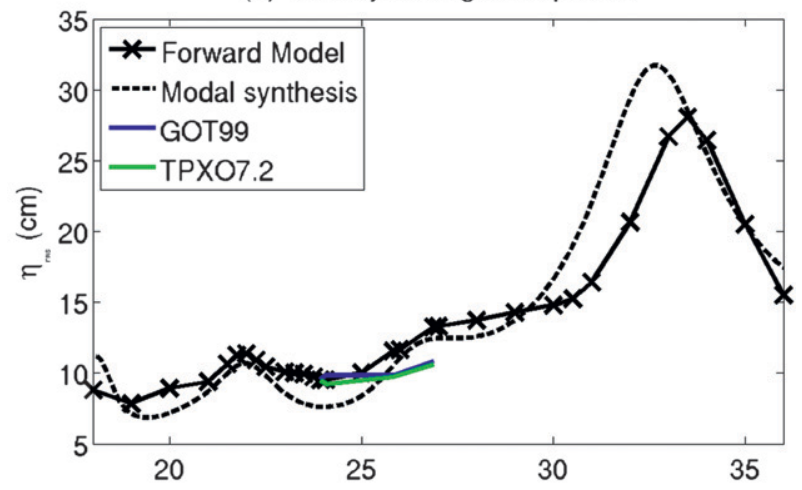

(b) Shape factor of free oscillations

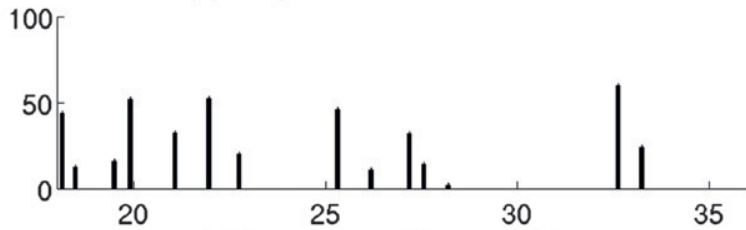

(c) Decay time of free oscillations



FIG. 3. (a) Globally and temporally averaged $\eta_{\text {rms }}$ values (cm) computed over the deep ocean (water depths $1000 \mathrm{~m}$ and greater) equatorward of $66^{\circ}$ in simulations of the forward and modal synthesis models with $K_{1}$-like astronomical forcing and realistic ocean geometry but with a varying forcing period (equivalently, varying frequency). Additionally, results from the satellite-altimetryconstrained models TPXO7.2 and GOT99, in which $Q_{1}, O_{1}$, and $P_{1}$ results are rescaled as if their astronomical forcing amplitude were equal to that of $K_{1}$, are shown. (b) Shape factor $\left\langle\mathbf{F}_{\text {tide }}, \hat{\mathbf{x}}_{k}\right\rangle$ and (c) the decay time $T_{D}=1 /\left(2 \omega_{1}\right)$ of free oscillations in the diurnal period range. [See Zahel and Müller (2005), and note that in that paper modal frequencies are denoted by the symbol $\sigma$ instead of $\omega]$.

(scaled) $P_{1}$, but is not as close for (scaled) $O_{1}$ and (scaled) $Q_{1}$.

In Figs. $3 \mathrm{~b}$ and $3 \mathrm{c}$ we show the shape factors and decay times of all free oscillations in the diurnal spectrum, as determined by Müller (2008). Obviously not every free oscillation yields a peak in the frequency sweep. Only if friction is low (i.e., the decay time is large) and the shape factor is large (which corresponds to a high spatial coherence of the corresponding adjoint free oscillation with the forcing field) is a peak likely to be generated. Decay times are apparently the most important factor, since the 26.20-h mode has a fairly low shape factor, but still yields a small peak in the frequency sweep. The
TABLE 3. Second, third, and fourth columns: Periods, astronomical forcing amplitudes, and Love number combination $1+k_{2}-h_{2}$, respectively, for the four largest diurnal constituents listed in the first column. Fifth column: scale factors [the ratio of the value of $A\left(1+k_{2}-h_{2}\right)$ for $K_{1}$ to that of the constituent in question] used to plot the TPXO7.2 and GOT99 results on diurnal constituents in Figs. 3, 7, and 8 .

\begin{tabular}{ccccl}
\hline \hline Constituent & $\begin{array}{c}\text { Period } \\
(\mathrm{h})\end{array}$ & $\begin{array}{c}\text { Astronomical forcing } \\
\text { amplitude }(\mathrm{cm})\end{array}$ & $\begin{array}{c}\text { Love number } \\
\text { combination }\end{array}$ & $\begin{array}{c}\text { Scale } \\
\text { factor }\end{array}$ \\
\hline$K_{1}$ & 23.9345 & 14.1565 & 0.736 & 1 \\
$P_{1}$ & 24.0678 & 4.6848 & 0.706 & 3.1502 \\
$O_{1}$ & 25.8192 & 10.0661 & 0.695 & 1.4893 \\
$Q_{1}$ & 26.8684 & 1.9273 & 0.695 & 7.7786 \\
\hline
\end{tabular}

32.64-h mode has the largest decay time and shape factor, explaining its dominance in the frequency sweep.

The amplitudes and phases of the 21.97-, 26.20-, and 32.64-h normal modes are displayed in Fig. 4. The 21.97- and 26.20-h modes display a strong signature in the North Pacific, while the 32.64-h mode displays a strong signature of an Antarctic Kelvin wave.

The largest peak in Fig. 3a corresponds to the free oscillation with the 32.64-h period, which represents the first-order Antarctic Kelvin wave and Kelvin wave propagating along the North Pacific coasts (Figs. $4 \mathrm{e}$ and 4f). This free oscillation is one of the major contributors to the main diurnal tides (Müller 2008), although the 32.64-h mode lies farther from the diurnal band than many other modes shown in Figs. $3 \mathrm{~b}$ and $3 \mathrm{c}$. The importance of this free oscillation stems from its large damping time of about $54 \mathrm{~h}$ and its large shape factor. The two modes at 21.97 and $26.20 \mathrm{~h}$, seen as peaks in the frequency sweep, are also important in the modal synthesis of diurnal tides. All three modes shown in Fig. 4 have a signature of the Antarctic Kelvin wave, but only the 26.20- and 32.64-h modes significantly contribute to the tidally forced one. The $32.64-\mathrm{h}$ mode has $49 \%$ of its total energy in the Southern Ocean, while the 21.97 and 26.20 -h modes have $17 \%$ and $24 \%$ of their energy in the Atlantic Ocean, respectively, and $58 \%$ of their total energy in the Pacific Ocean. A detailed discussion of the features of all modes used for the modal synthesis model and their contribution to the diurnal tides can be found in Müller (2008, 2009).

A conclusive result is that the globally averaged $\eta_{\mathrm{rms}}$ values displayed in Fig. 3a are quite consistent between the forward ocean tide model and the modal synthesis model. The peaks near 22 and $33 \mathrm{~h}$, for instance, nearly coincide between the two models.

\section{b. Sensitivity to zonal structure of forcing}

In this subsection we conduct simulations with the forward ocean tide model and the global free oscillations 
(a) 21.97 Hour Mode

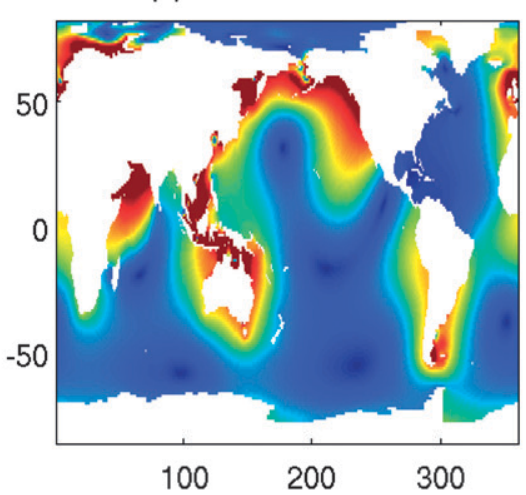

(c) 26.20 Hour Mode



(e) 32.64 Hour Mode

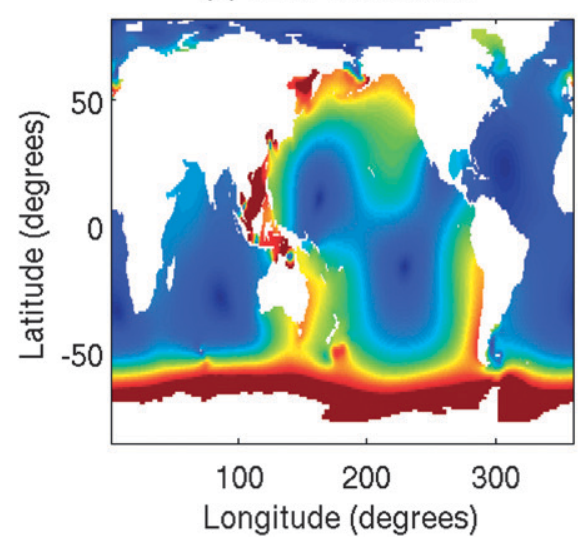

(b) 21.97 Hour Mode
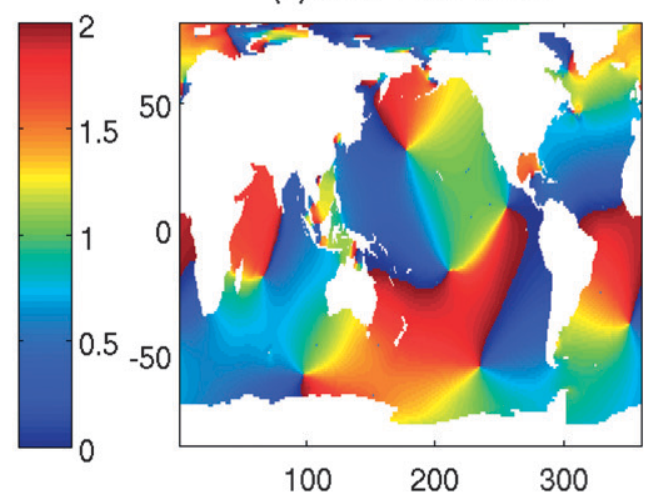

(d) 26.20 Hour Mode
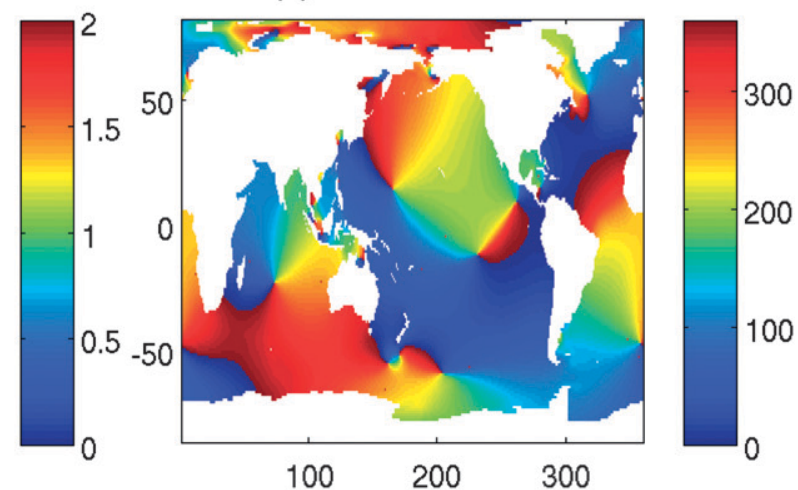

(f) 32.64 Hour Mode

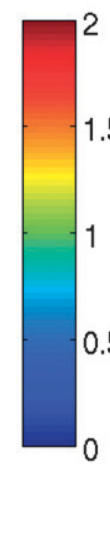

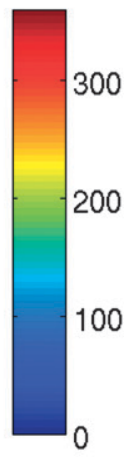

FIG. 4. Amplitude and Greenwich phase $\left({ }^{\circ}\right)$, respectively, of tidal elevations for the (a),(b) 21.97-h mode, for the (c),(d) 26.20-h mode, and for (e),(f) the 32.64-h mode. Amplitude and phase are normalized as in Müller (2008).

model, with the same basin geometry and topography as in the nominal solution but in which the zonal structure of the astronomical diurnal tidal forcing is varied. We set

$$
\eta_{\mathrm{EQ}}=\left(1+k_{2}-h_{2}\right) A_{\text {astronomical }} \sin (2 \phi) \cos (\omega t+N \lambda),
$$

where $N$, the zonal wavenumber, takes on values of 0,1 , $2,3,4,5,8$, and 16 (recall that $N=1$ is the nominal realistic case). As in section $4 \mathrm{a}$, maps of the tidal amplitudes for these experiments are not shown for the sake of brevity. All of them differ (in pattern as well as magnitude) from the amplitude map of the nominal 
(a) Globally averaged amplitude



(b) Shape factor

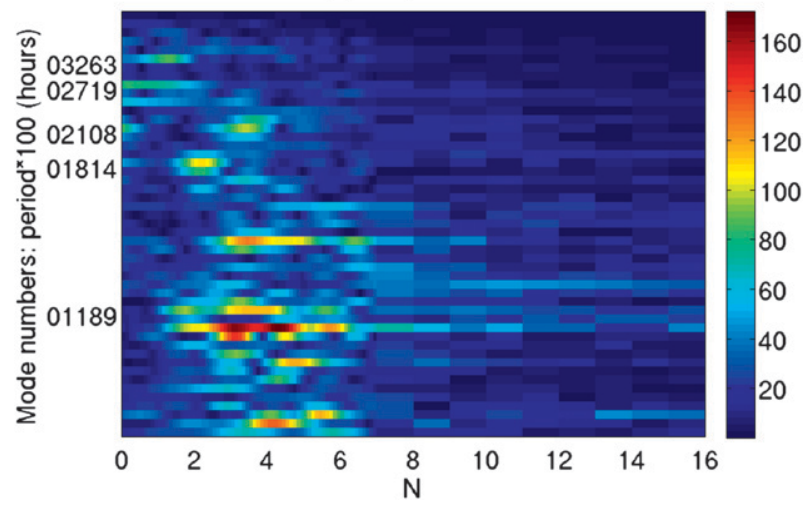

FIG. 5. (a) Globally and temporally averaged $\eta_{\text {rms }}$ values (cm), computed over latitudes equatorward of $66^{\circ}$ in water depths exceeding $1000 \mathrm{~m}$ (open ocean) and in depths shallower than $1000 \mathrm{~m}$ (shelf), in simulations with astronomical forcing like that of $K_{1}$ but with a varying zonal wavenumber $N$ in Eq. (15). The extra vertical line represents the realistic case $(N=1)$. (b) Shape factors $\left\langle\mathbf{F}_{\text {tide }}, \hat{\mathbf{x}}_{k}\right\rangle$ for the free oscillations in the period range from 10 to $80 \mathrm{~h}$ plotted vs $N$.

simulation, with the $N=16$ experiment displaying the largest discrepancies.

The $\eta_{\mathrm{rms}}$ values, in the open ocean and in the shelf, are plotted versus $N$ in Fig. 5a. The vertical line in Fig. 5a marks the value of $N$ corresponding to the realistic case $(N=1)$. As can be seen in Fig. 5a, predictions from the modal synthesis model match general trends seen in the forward ocean tide model results, although there are clear discrepancies. Figure 5b displays the dependence of the shape factor on the zonal wavenumber for modes with periods between 10 and $80 \mathrm{~h}$. This figure clearly shows clusters of large shape factors between $N=1$ and 4 , which aligns with the strong oceanic response seen in Fig. 5a.

\section{c. Sensitivity to meridional structure of forcing}

In this subsection we investigate the sensitivity of tidal elevations to meridional structure in the astronomical diurnal tidal forcing, again using the forward ocean tide model and the modal synthesis model. As in sections $4 \mathrm{a}$ and $4 \mathrm{~b}$, each simulation in this section is conducted with realistic basin geometry and topography. The astronomical forcing of the second-degree diurnal tides is proportional to the spherical harmonic

$$
\begin{aligned}
Y_{2}^{1}(\phi, \lambda) & =N_{2}^{1} \sin (2 \phi) e^{i \lambda} \text { and } \\
N_{2}^{1} & =-\frac{1}{4} \sqrt{\frac{15}{2 \pi}}
\end{aligned}
$$

where $N_{2}^{1}$ is a normalization constant (Arfken and Weber 2001). This normalization constant ensures that integrating $\left|Y_{2}^{1}(\phi, \lambda)\right|^{2}$ over a unit sphere yields unity. We rewrite the gravitational forcing in Eq. (9) as

$$
\eta_{\mathrm{EQ}}=\left(1+k_{2}-h_{2}\right) A_{\text {astronomical }} \frac{1}{N_{2}^{1}} \Re\left[Y_{2}^{1}(\phi, \omega t+\lambda)\right],
$$

where $\Re\left[Y_{2}^{1}(\phi, \omega t+\lambda)\right]$ represents the real portion of $Y_{2}^{1}(\phi, \omega t+\lambda)$. To vary the meridional structure of the astronomical diurnal tidal forcing, we construct tidal potentials of degree $l=1,2,3,4$, and 5 , using the associated spherical harmonics of degree $l$ :

$$
\eta_{\mathrm{EQ}}=\left(1+k_{2}-h_{2}\right) A_{\text {astronomical }} \frac{\beta_{l}^{1}}{N_{2}^{1}} \Re\left[Y_{l}^{1}(\phi, \omega t+\lambda)\right] .
$$

Here $\beta_{l}^{1}$ is a normalization constant guaranteeing that the rms value of the equilibrium tidal forcing averaged over the oceanic area is the same for each value of $l$ as for $K_{1}$ forcing $(l=2)$. We enforce this criterion so that discrepancies between the $l \neq 2$ forward ocean tide (and modal synthesis) experiments and the nominal $l=2$ cases are due to changes in the spatial structure and not to the magnitude of the astronomical forcing averaged over the area of the World Ocean. Thus, we set

$$
\beta_{l}^{1}=\sqrt{\frac{F_{2}^{1}}{F_{l}^{1}}},
$$

where $F_{l}^{1}$ is defined by

$$
F_{l}^{1}=\iint_{\text {ocean area }}\left|Y_{l}^{1}\right|^{2} d A .
$$

The values of $\beta_{l}^{1}$ deviate by $4 \%$ or less from unity for all values of $l$ and for both the forward and modal synthesis models.

As shown in Fig. 6, the forward ocean tide model and modal synthesis model both display a peak in response 
(a) Globally averaged amplitude

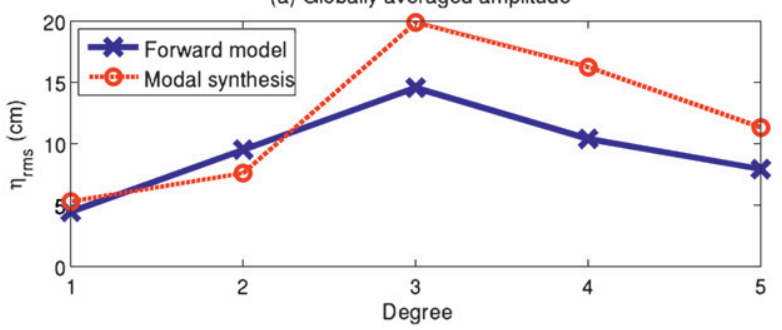

(b) Shape factor

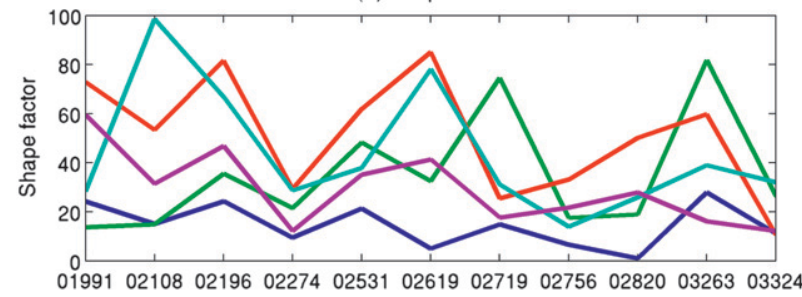

(c) Weighting coefficient



FIG. 6. (a) Globally and temporally averaged $\eta_{\text {rms }}$ values $(\mathrm{cm})$, computed over latitudes equatorward of $66^{\circ}$ and in water depths exceeding $1000 \mathrm{~m}$ (open ocean), in simulations with astronomical forcing like that of $K_{1}$ but with a varying degree $l$ of the spherical harmonic in Eq. (19). (b) Shape factor and (c) weighting coefficients $a_{k}$ plotted as a function of mode number, that is, the period of the free oscillation in hours multiplied by 100 . Colored lines in (b),(c) represent the solutions for different degree of the spherical harmonic forcing function.

for degree $l=3$. To help us understand which modes are responsible for this strong response to third-degree forcing, in Figs. $6 \mathrm{~b}$ and $6 \mathrm{c}$ we plot the shape factors and weighting coefficients [see Eq. (6) and Eq. (8)] of free oscillations in the diurnal spectrum for degrees $l=1,2$, 3,4 , and 5 , respectively. These figures show that the peak seen in Fig. 6a for $l=3$ is primarily produced by the 21.97- and 26.20-h modes. The adjoint counterparts of these modes fit best to third degree-shaped forcing, and thus, combined with their long decay times (see Fig. 3c) and near resonance, they dominate with large weighting coefficients. However, when the tidal forcing is second degree, the shape factors of the 21.97- and 26.20-h modes are reduced by a factor of $\sim 2$. This implies that the second-degree tidal forcing is not as effective as the third-degree tidal forcing, for tides of diurnal frequency.

The results presented here on the sensitivity of diurnal tides to meridional forcing structure are consistent with results of previous studies of the third-degree diurnal tides in the North Atlantic (Cartwright 1975; Ray 2001). In these previous studies the relatively large $M_{1}$ tide observed in the North Atlantic was explained by the correlation (shape factors) of free oscillations with the periods of 23.7 and $25.7 \mathrm{~h}$, which were computed by Platzman et al. (1981). The free oscillations with these periods also correlated better with third-degree forcing than with second-degree forcing. Furthermore, the spatial structure of the 26.20-h mode of the present study is similar to that of the 25.7-h mode of Platzman et al. (1981), with two amphidromes located in the North and South Atlantic (see Fig. 4).

To summarize the results of sections $4 a, 4 b$, and $4 c$, the modal synthesis model predicts the sensitivity of the forward ocean diurnal tide model to the frequency, zonal structure, and meridional structure of the astronomical tidal forcing with a fairly high degree of skill. This adds to evidence, previously accumulated by other means, that open-ocean tides can be reasonably thought of as a superposition of damped oscillatory normal modes. We next turn our attention to the resonance of coastal diurnal tides and to the impact on open-ocean diurnal tides of blocking out regions of prominent coastal diurnal tides.

\section{d. Coastal tidal sensitivity to forcing frequency}

We now use the frequency sweep simulations of the forward tide model to better understand the coastal diurnal tides. In Figs. 7 and 8 we show the tidal amplitudes and phases at six locations of large coastal diurnal tides, plotted against the forcing period $2 \pi / \omega$ in the frequency sweep. The amplitudes and phases in Figs. 7 and 8 are taken from specific model gridpoints, given by the latitudes and longitudes listed in the titles of the various subplots. As in the open-ocean results shown in Fig. 3a, the coastal results in Figs. 7 and 8 include rescaled TPXO7.2 and GOT99 results. As with the open-ocean results, discrepancies between the forward ocean tide model and the altimetry-constrained models (and between the two altimetry-constrained models themselves) are clearly seen. However, as the forcing period varies, the forward ocean tide model usually exhibits the same trends as the satellite-constrained models.

Having shown that the forward model has some degree of skill in reproducing the frequency sensitivity in coastal zones, we now examine coastal resonance. The coastal locations in Figs. 7 and 8 all display peaks in their amplitude values somewhere within the period band of 22-33 h. For example, in the Sea of Okhotsk (Fig. 7a) a peak is seen near $22 \mathrm{~h}$, while in Bristol Bay (Fig. 7c) broad peaks appear near both 22 and $33 \mathrm{~h}$. The Gulf of Tonkin (Fig. 7e) and Ross Sea (Fig. 8e) peak near 33 h. 
(a) Sea of Okhotsk - $61^{\circ} \mathrm{N}, 163.5^{\circ} \mathrm{E}$ (b) Sea of Okhotsk -- $61^{\circ} \mathrm{N}, 163.5^{\circ} \mathrm{E}$
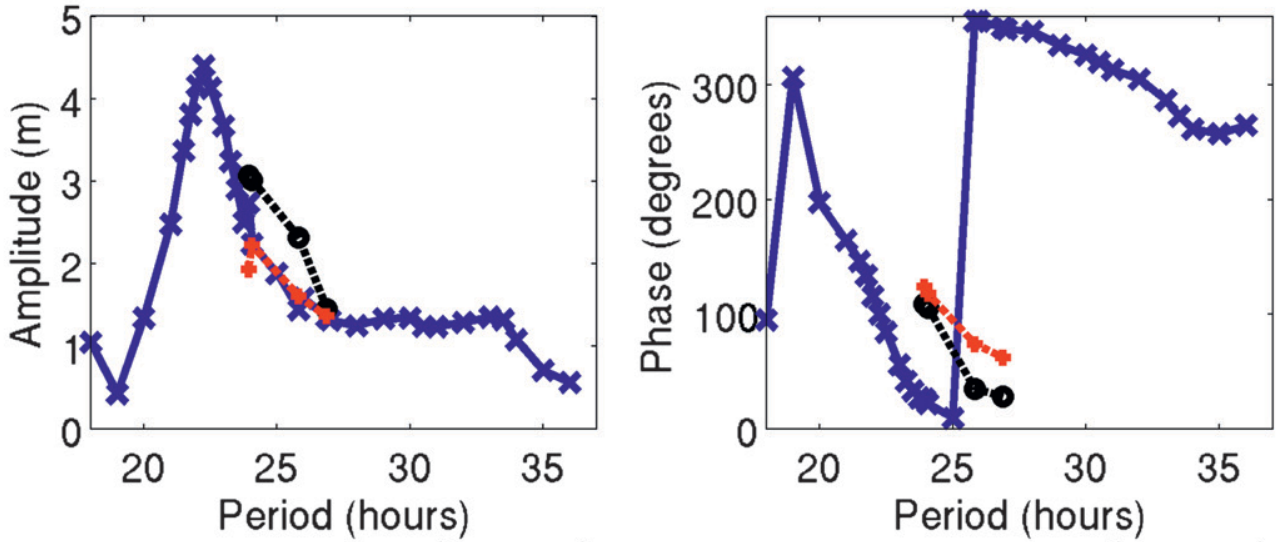

(c) Bristol Bay -- $58.5^{\circ} \mathrm{N}, 202^{\circ} \mathrm{E}$

(d) Bristol Bay -- $58.5^{\circ} \mathrm{N}, 202^{\circ} \mathrm{E}$
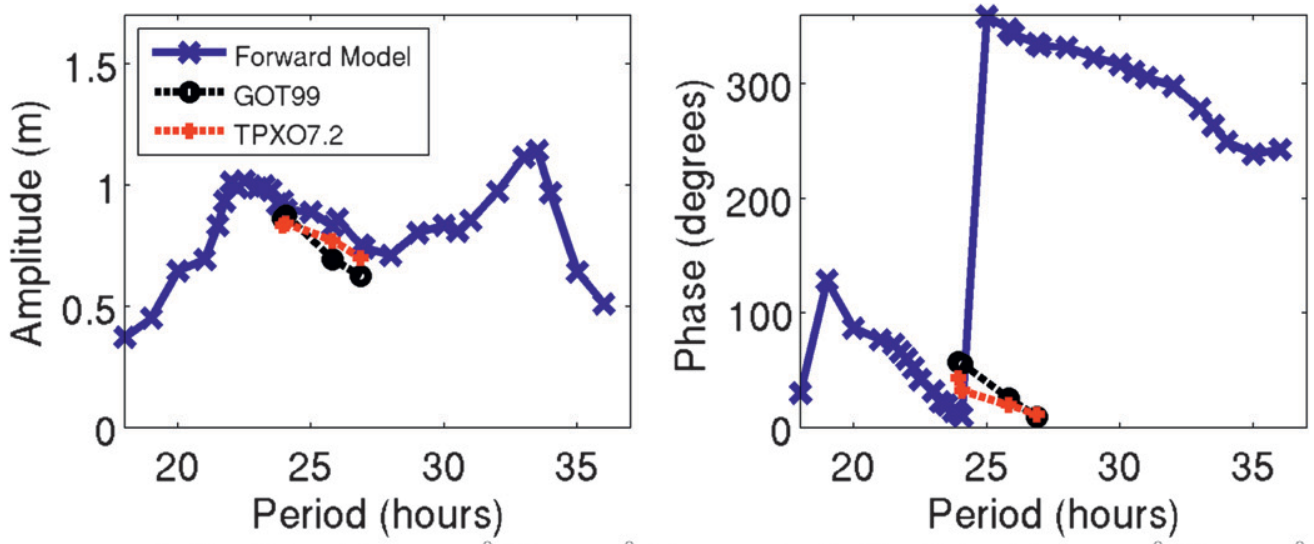

(e) Gulf of Tonkin -- $21^{\circ} \mathrm{N}, 109^{\circ} \mathrm{E}$

(f) Gulf of Tonkin -- $21^{\circ} \mathrm{N}, 109^{\circ} \mathrm{E}$
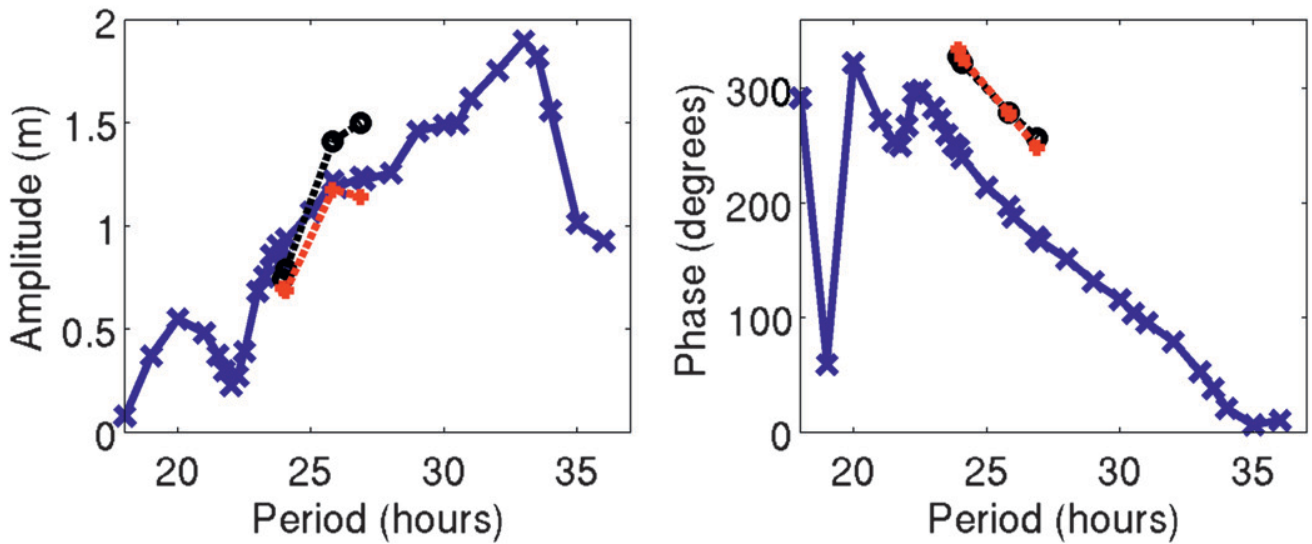

FIG. 7. Amplitude (m) and Greenwich phase $\left(^{\circ}\right)$ of tidal elevations in the Sea of Okhotsk, Bristol Bay, and Gulf of Tonkin in forward tide model experiments with astronomical forcing like that of $K_{1}$ but with a varying forcing period (equivalently, varying frequency). Results from the satellite-altimetry-constrained models TPXO7.2 and GOT99 in which $Q_{1}, O_{1}$, and $P_{1}$ results are rescaled as if their equilibrium forcing amplitude were equal to that of $K_{1}$, are also shown. Results are taken from specific model grid points, identified by the latitudes and longitudes in the titles of each subplot. 
(a) Sahul Shelf -- $6^{\circ} \mathrm{S}, 138^{\circ} \mathrm{E}$

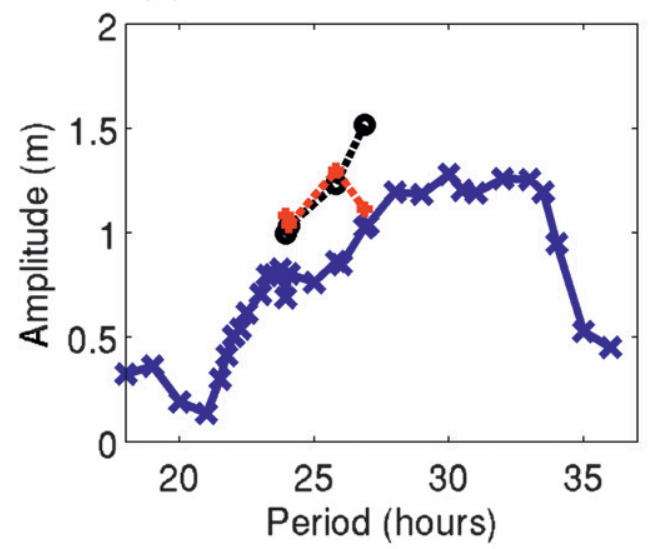

(c) Northwest Australia -- $14^{\circ} \mathrm{S}, 129.5$

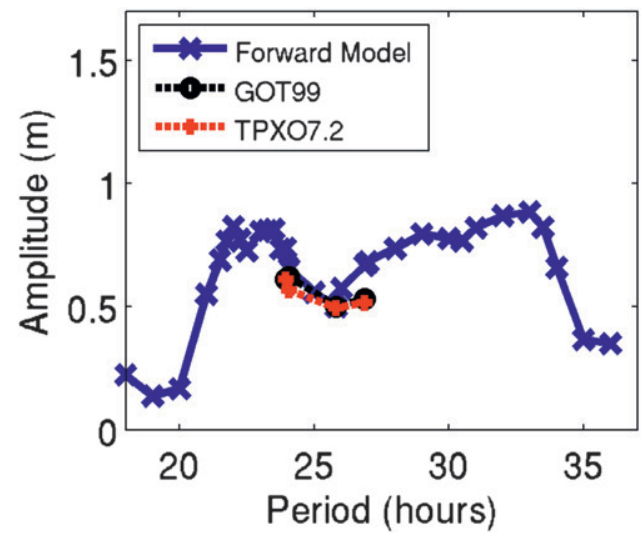

(e) Ross Sea -- $73.5^{\circ} \mathrm{S}, 178^{\circ} \mathrm{E}$

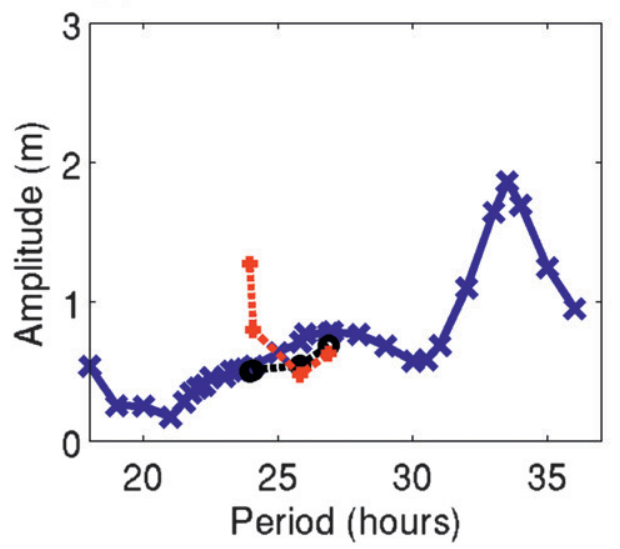

(b) Sahul Shelf $-6^{\circ} \mathrm{S}, 138^{\circ} \mathrm{E}$

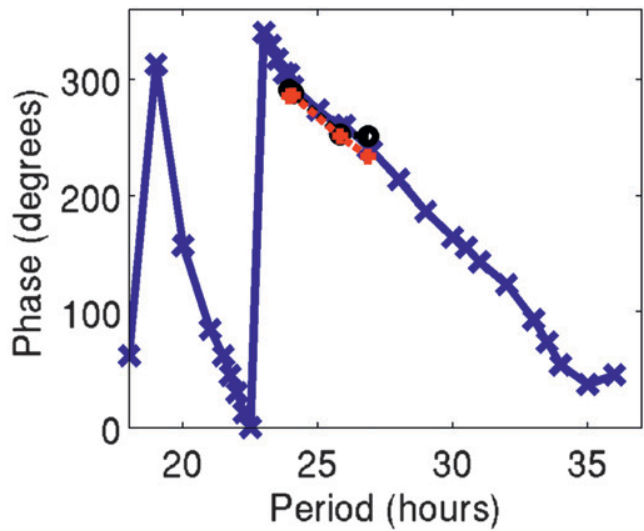

E (d) Northwest Australia -- $14^{\circ} \mathrm{S}, 129.5^{\circ} \mathrm{E}$

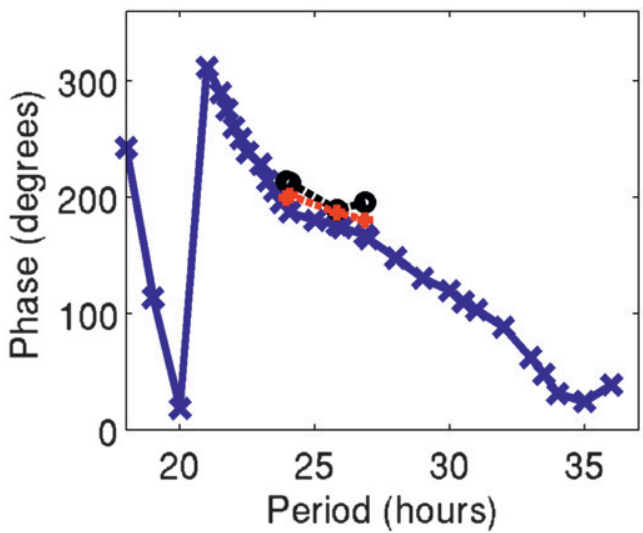

(f) Ross Sea -- $73.5^{\circ} \mathrm{S}, 178^{\circ} \mathrm{E}$



FIG. 8. As in Fig. 7, but for the Sahul Shelf, northwest Australia, and Ross Sea.

The peaks near periods of 22 and $33 \mathrm{~h}$ are driven by the global free oscillations with periods of 21.97 and $32.64 \mathrm{~h}$. Both are major components of the diurnal tides.

The spectral peaks in the coastal regions, and their consistency with the frequencies of the major free oscillations for diurnal tides, reflect that the tides are driven in these regions by global free oscillations. The global free oscillations represent coupled shelf-open ocean resonances. We next attempt to separate, to the extent that we can, the coastal resonances from the global free oscillations. To do this we extend the admittance analysis of Garrett (1972) to selected coastal 
regions of interest, using the frequency sweep simulations from the forward ocean tide model.

To identify the presence of coastal resonance, we investigate the amplitude-phase relationships between the "inside" coastal system and the corresponding "outside" open-ocean system. Garrett (1972) developed an approach using three semidiurnal tidal constituents inside and outside of the Gulf of Maine-Bay of Fundy system and derived the coastal resonance period of $13.3 \mathrm{~h}$, for this system. A particular feature of his method is that he acknowledged the much stronger forced $M_{2}$ compared to the other two considered tidal constituents, that is, $S_{2}$ and $N_{2}$. Thus, nonlinearities in the tidal response induced by nonlinear bottom friction were explicitly included. In the present study, we will use the frequency sweep simulations. Since each simulation within the frequency sweep consists of one constituent only, we can neglect these considerations of nonlinear response, meaning that the value of the parameter " $c$ " in Garrett (1972) is one.

The tidal amplitudes $A_{\text {in }}$ and $A_{\text {out }}$ and phases $\phi_{\text {in }}$ and $\phi_{\text {out }}$ inside and outside the coastal system are written as amplitude ratios $R$ and phase differences $\Phi$ :

$$
\begin{aligned}
& R(\omega)=\frac{A_{\text {in }}(\omega)}{A_{\text {out }}(\omega)} \text { and } \\
& \Phi(\omega)=\phi_{\text {in }}(\omega)-\phi_{\text {out }}(\omega),
\end{aligned}
$$

where $\omega$ represents the frequencies of the frequency sweep experiments. We further define a "reference" frequency $\omega_{\text {ref }}$ [in Garrett (1972) the $S_{2}$ tidal frequency], and write

$$
\hat{y}(\omega)=\frac{R(\omega)}{R\left(\omega_{\text {ref }}\right)} \exp \left\{i\left[\Phi(\omega)-\Phi\left(\omega_{\text {ref }}\right)\right]\right\}
$$

We assume now that the response of the system is in the considered frequency range of one single resonantly forced mode and write the response of the coastal system as (Garrett 1972)

$$
C\left(\omega, \omega_{0}, Q, c\right)=\left[\frac{\omega_{0}-\omega}{\omega}-0.5 i(c Q)^{-1}\right]^{-1}
$$

with the unknown resonance frequency $\omega_{0}$, damping factor $Q$, and nonlinearity constant $c$ (as discussed above, in our case $c=1$ ). Garrett (1972) argues that we can write

$$
\frac{C\left(\omega, \omega_{0}, Q, c\right)}{C\left(\omega_{\text {ref }}, \omega_{0}, Q, c\right)}=\hat{y}\left(\omega, \omega_{0}, Q\right)
$$
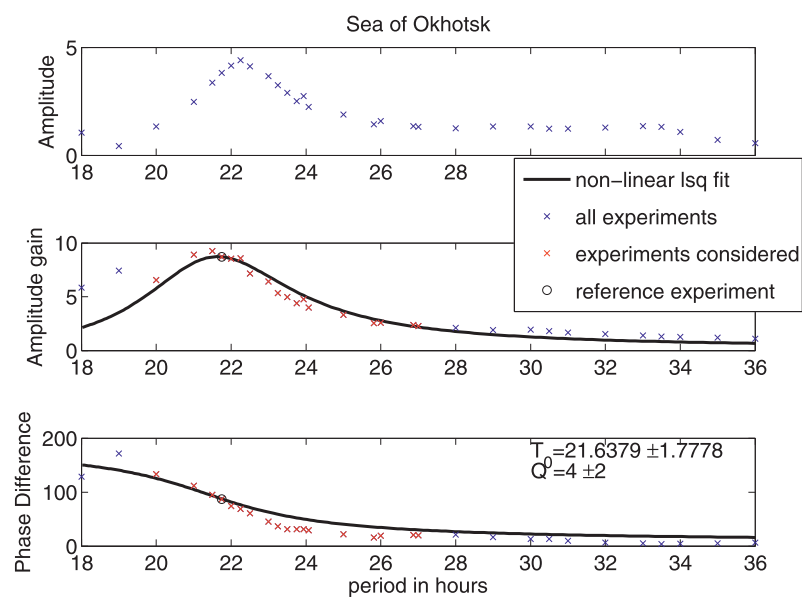

FIG. 9. The Garrett (1972) method applied to the frequency sweep experiments and Sea of Okhotsk. The inside point is located at $\left(61^{\circ} \mathrm{N}, 163.5^{\circ} \mathrm{E}\right)$, and the outside grid point at $\left(53.5^{\circ} \mathrm{N}, 160.5^{\circ} \mathrm{E}\right)$. (top) The amplitude at the inside Sea of Okhotsk point vs forcing period. (middle) The amplitude of the response function [see Eq. (25)] of the coastal system. (bottom) The phase of the response function. Blue crosses depict all frequency sweep experiments, red crosses show experiments used for the resonance fit, and the circle denotes the frequency chosen as the reference frequency.

and thus obtain estimates of the resonance frequency $\omega_{0}$ and the $Q$ factor. Since this equation is only valid for a single mode resonance, we have to manually select a particular frequency range around $\omega_{0}$. In Fig. 9 a nonlinear least squares fit of Eq. (26) has been performed for the Sea of Okhotsk. All points shown in red have been considered for the fit and the values including their $95 \%$ confidence intervals are $T=\omega_{0} /(2 \pi)=21.7 \pm 1.8 \mathrm{~h}$ and $Q=4 \pm 2$.

This result clearly reflects that the Sea of Okhotsk itself is resonant with a resonance period of $\sim 22 \mathrm{~h}$. For all regions shown in Figs. 7 and 8, the aforementioned analysis has been applied, and we find that the Sahul Shelf and Gulf of Tonkin harbor coastal resonances with respective natural oscillation periods of about 27 and $29 \mathrm{~h}$ (not shown here for the sake of brevity), as well, whereas the other coastal regions in Figs. 7 and 8 cannot be shown to be resonant by this technique. Application of the admittance analysis to the most important region for semidiurnal tides identified in $\mathrm{AKG}$ - the Hudson Strait-demonstrates that it is also resonant (not shown here for the sake of brevity).

\section{e. Back effect of shelf upon open ocean in blocking experiments}

We now explore the back effect of coastal diurnal tides on open-ocean diurnal tides in forward ocean tide model experiments with basin geometries that are realistic except for the fact that coastal regions of interest 
are blocked off one at a time. Figures $10 \mathrm{~b}, \mathrm{e}-15 \mathrm{~b}, \mathrm{e}$ display elevation amplitudes in $K_{1}$ experiments in which portions of the Sea of Okhotsk, Bristol Bay, the Bering Sea, the Sahul Shelf, the northwest Australian Shelf, the Ross Sea, the Gulf of Tonkin, the west coast of South America, the Bay of Bengal, Bass Strait, and the Gulf of Mexico, have been blocked out one at a time. The Blocked Large Sea of Okhotsk region (Fig. 10b) blocks off the majority of the sea in which depths are shallower than $1000 \mathrm{~m}$. The Blocked Small Sea of Okhotsk region (Fig. 10e) is defined by the region where diurnal tidal elevations within the sea exceed $2.0 \mathrm{~m}$ in the nominal simulation. The ocean gridpoints omitted in the Blocked Sahul Shelf simulation include the gridpoints omitted in the Blocked Australian Shelf simulation. For comparison, Figs. 10a,d-15a,d show the amplitudes in these regions for the nominal experiment (also referred to as "unblocked"). In each of the blocked experiments a large portion of the coastal region in question is removed so that features of interest such as previously large coastal tides are now absent. Several of the regions were chosen for having large amplitude coastal diurnal tides, but some regions were chosen for other reasons. The Bering Sea has large diurnal tidal dissipation (Fig. 2), but relatively small diurnal tidal amplitudes (Fig. 11d). The west coast of South America, Bay of Bengal, Bass Strait, and Gulf of Mexico were chosen as "control" regions, in which neither the coastal diurnal tidal elevations or dissipation are particularly strong. ${ }^{1}$ The maximum depth of the grid points blocked out never exceeds $1000 \mathrm{~m}$, except in the Blocked Ross Sea experiment. In that experiment, we enforce the criterion that depths of removed grid points not exceed $2000 \mathrm{~m}$; in the nominal simulation, the Ross Sea possesses large tidal elevations in the depth range between 1000 and $2000 \mathrm{~m}$.

We emphasize the impact of blocking by also showing the difference in elevation amplitudes between the blocked and unblocked simulations (Figs. 10c,f-15c,f). In all of the blocked simulations, amplitude differences of $5-10 \mathrm{~cm}$ or more are easily seen, and the blocking affects the tides on basinwide and even global scales. The globally averaged rms signals $\eta_{\mathrm{rms}}$ for the blocking simulations are shown in Table 4. In most of the blocked

\footnotetext{
${ }^{1}$ We thank an anonymous reviewer for suggesting additional blocking experiments, in the Bering Sea and in some "control" regions, beyond what was presented in the original manuscript. It should be noted that the diurnal tides in the Gulf of Mexico are considerably stronger than the very weak diurnal tides in the North Atlantic. Thus, compared to other nearby locations, the Gulf of Mexico diurnal tides are not small.
}

simulations, $\eta_{\mathrm{rms}}$ increases from its value in the nominal simulation, as in the semidiurnal simulations of AKG.

Other measures of the alterations to the global tide caused by removing regions of large coastal tides can be computed. A measure that accounts for changes in phase (which can be on the order of $50^{\circ}-100^{\circ}$ but are not shown for the sake of brevity), as well as in amplitude, is the perturbation

$$
P=\sqrt{\left[\iint\left(\eta_{\text {blocked }}-\eta_{\text {nominal }}\right)^{2} d A\right] / \iint d A},
$$

where $\eta_{\text {blocked }}$ is the elevation in the blocked simulation, $\eta_{\text {nominal }}$ is the elevation in the nominal simulation, and the area average is again computed over water depths exceeding $1000 \mathrm{~m}$ and latitudes equatorward of $66^{\circ}$. Owing to the latter restriction, this diagnostic does not account for the largest changes seen in the Southern Ocean for the Blocked Ross Sea (Fig. 13c) simulation. The perturbations are also given in Table 4, and range from $0.68 \mathrm{~cm}$ in the Blocked Bass Strait simulation to $3.00 \mathrm{~cm}$ in the Blocked Large Sea of Okhotsk simulation.

The globally integrated dissipation rates calculated via Eq. (14) for the nominal and blocked simulations are also provided in Table 4. The global dissipation rate upon removal of specific coastal areas is sometimes larger but usually smaller than the global dissipation rate in the nominal simulation. This contrasts with the results of semidiurnal blocking simulations in AKG, which typically saw a rise in global dissipation rates with blocking.

In Table 4 we also show the area blocked out in each blocking simulation, and the perturbation to open-ocean diurnal tides per area blocked out. Thus we see, for instance, that the large perturbations in the Blocked Gulf of Mexico, Blocked Sahul Shelf, and Blocked Large Sea of Okhotsk simulations are in part due to the large areas blocked out in these simulations. The largest perturbation per area blocked out is seen in the Blocked Small Sea of Okhotsk simulation. In section $4 d$ the Sea of Okhotsk was shown to be resonant to diurnal tidal forcing. Thus, the large response to its blocking is consistent with the arguments of AKG and AG that the blocking of regions of resonant coastal tides strongly affects open-ocean tides. Also consistent with this notion is the fact that blocking the Hudson Strait, found here and in earlier papers (Arbic et al. 2007; Cummins et al. 2010) to be resonant to semidiurnal tidal forcing, has the largest effect on open-ocean semidiurnal tides of all regions examined in the blocking experiments of AKG. In further defense of the importance of coastal resonance in blocking experiments, we note that the 
(a) Unblocked Large Sea of Okhotsk

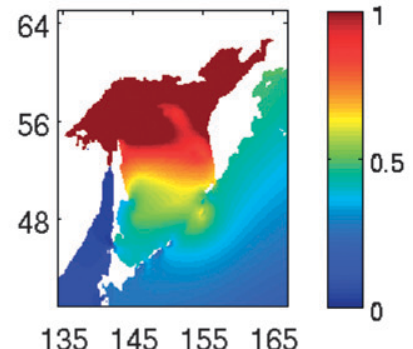

(b) Blocked Large Sea of Okhotsk

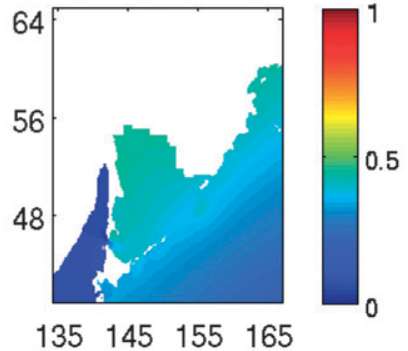

(d) Unblocked Small Sea of Okhotsk

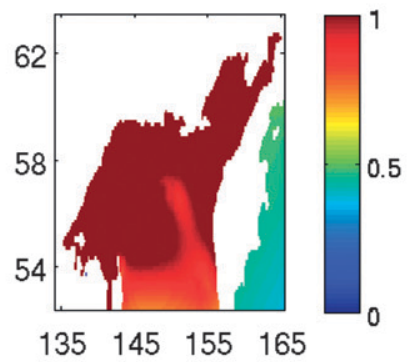

(e) Blocked Small Sea of Okhotsk

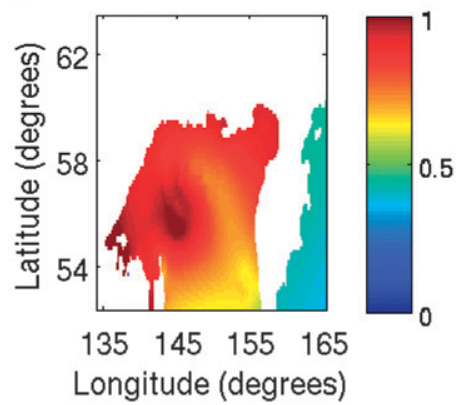

(c) Blocked Large Sea of Okhotsk - Unblocked

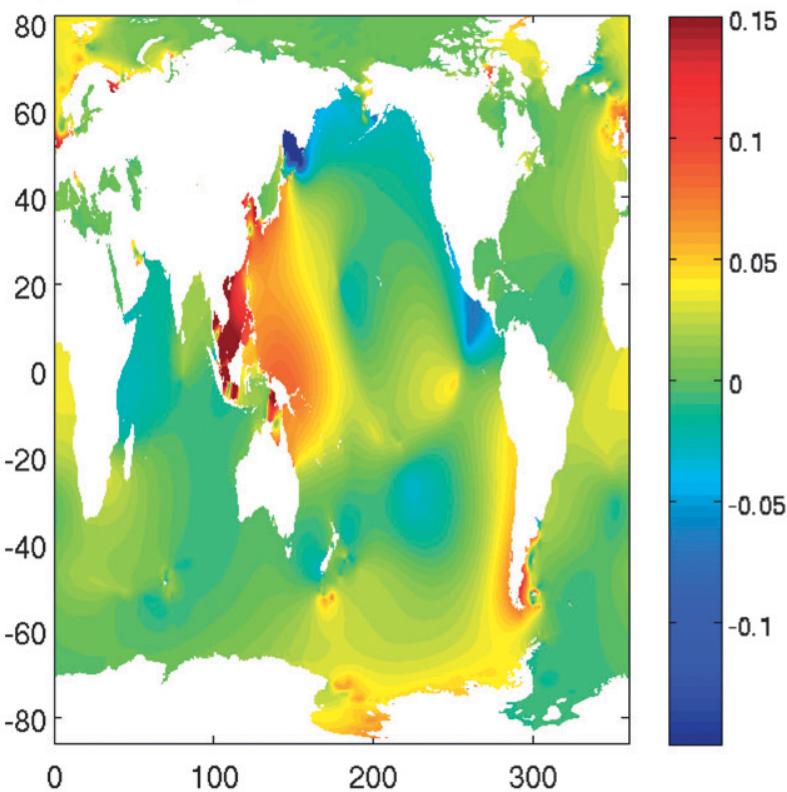

(f) Blocked Small Sea of Okhotsk - Unblocked

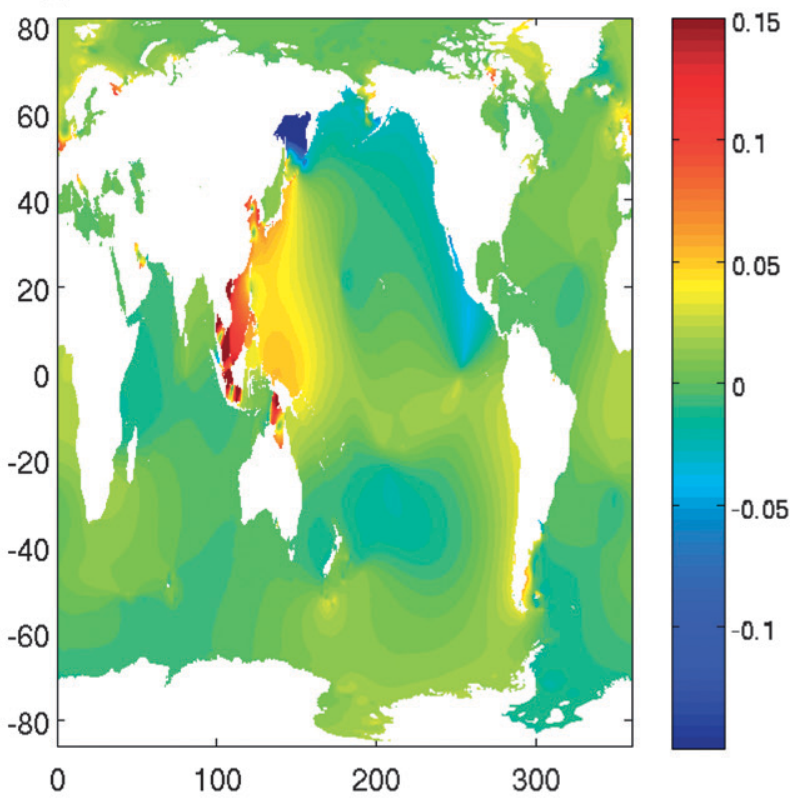

FIG. 10. (a),(b) The $K_{1}$ elevation amplitudes (m) in Sea of Okhotsk in (a) nominal (Unblocked) and (b) Blocked Large Sea of Okhotsk simulations. (c) Global map of $K_{1}$ elevation amplitude differences (m) between Blocked Large Sea of Okhtosk simulation and Unblocked simulation. (d)-(f) As in (a)-(c), but for Blocked Small Sea of Okhotsk simulation. Note change in scale between (c) and (a),(b) and between (f) and (d),(e).

blocking of the Sahul Shelf and Gulf of Tonkin, also found here to be resonant to diurnal forcing, both elicit large perturbations to open-ocean diurnal tides. Consistent as well with the simple models in $\mathrm{AG}$ and $\mathrm{AKG}-$ see for instance Eqs. (12)-(18) in AG-is the fact that blocking the Bering Sea, site of large diurnal tidal dissipation but relatively small diurnal elevations, elicits a large perturbation in open-ocean diurnal tides. Complicating these arguments, however, is the fact that blocking regions with neither large diurnal tides nor significant diurnal tidal dissipation-such as the west coast of South America, the Bay of Bengal, the Bass 
(a) Unblocked Bristol Bay

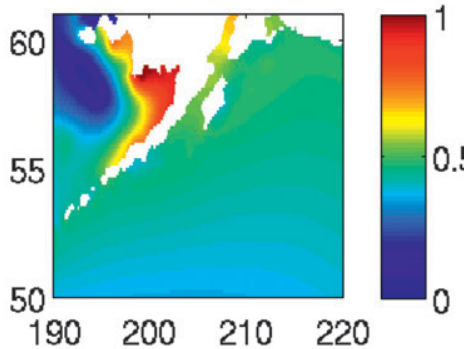

(c) Blocked Bristol Bay - Unblocked

(b) Blocked Bristol Bay
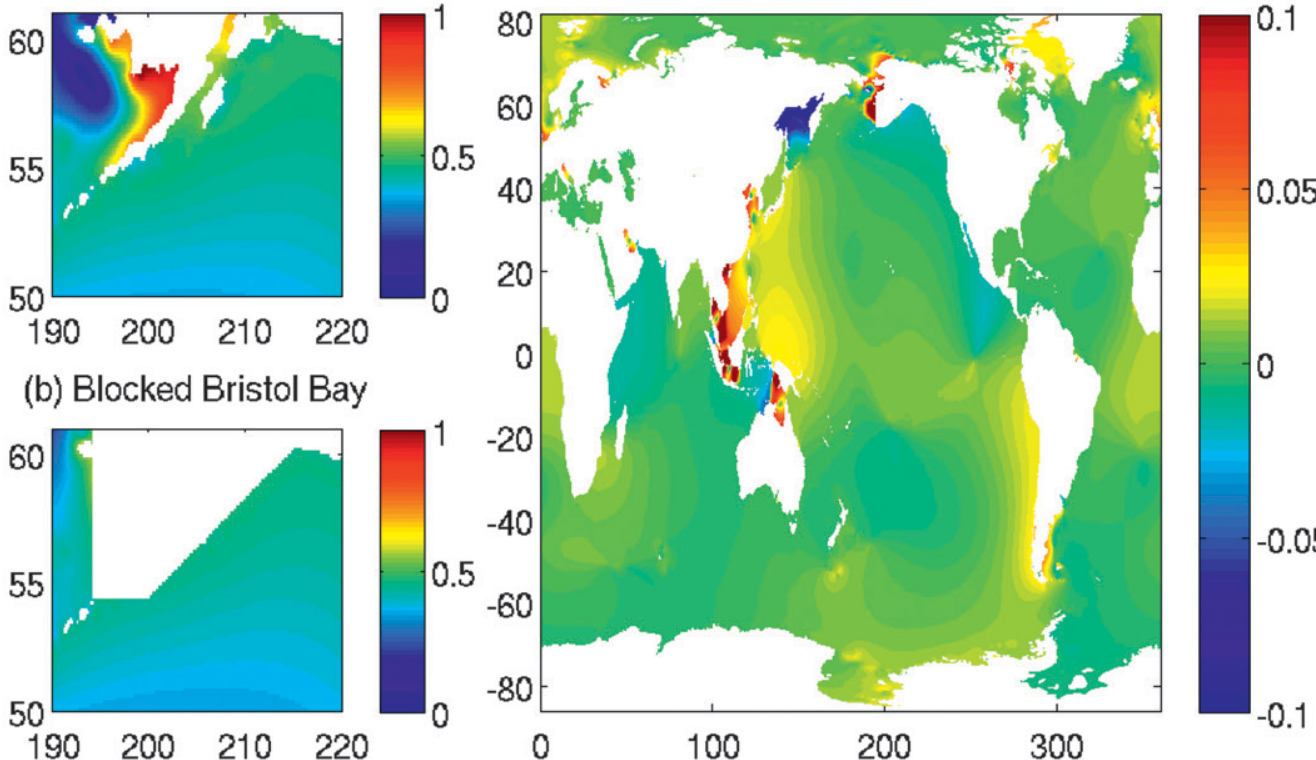

(d) Unblocked Bering Sea


(e) Blocked Bering Sea
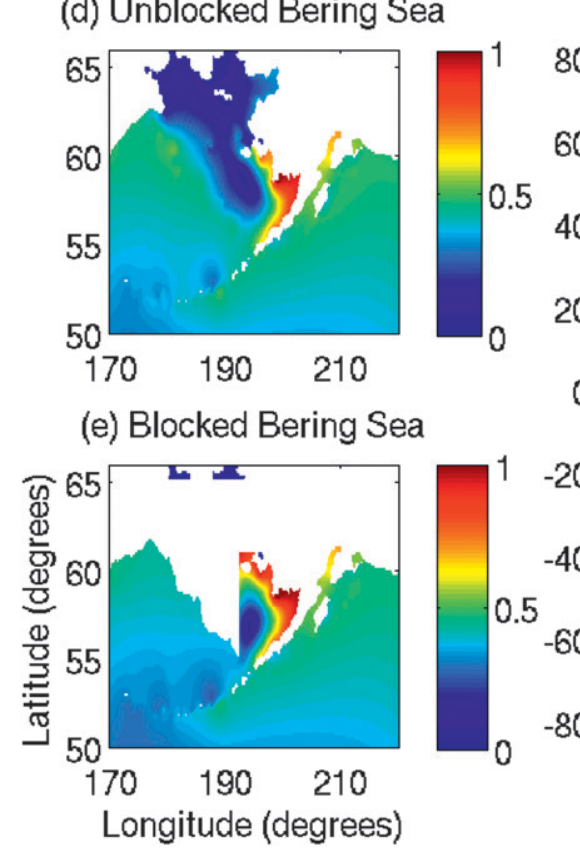

(f) Blocked Bering Sea - Unblocked

FIG. 11. As in Fig. 10, but for Blocked Bristol Bay and Blocked Bering Sea. For the difference maps the scale is reduced from that in Fig. 10.

Strait, and the Gulf of Mexico-also elicits large perturbations to open-ocean diurnal tides. It appears that strong resonance, strong dissipation, and simple coastal geometrical considerations irrespective of coastal resonances, are all capable of shaping the response of the open ocean to coastal tides.
We end this section with two notes. First, since clear deviations appear between our forward model and altimetry-constrained models in various coastal regions (i.e., the Sea of Okhotsk, Fig. 1), the results of the blocked experiments should be taken with some care. Second, in addition to performing the $K_{1}$ blocking 
(a) Unblocked Sahul Shelf

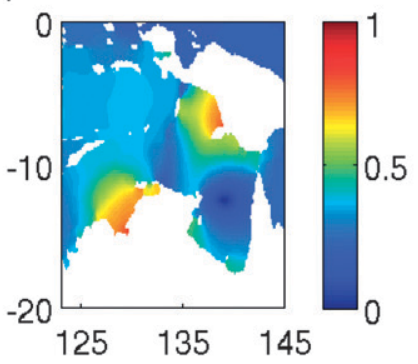

(b) Blocked Sahul Shelf

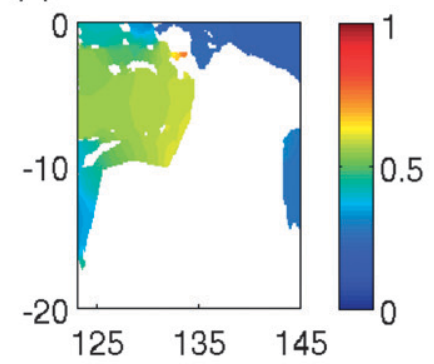

(d) Unblocked NW Australian Shelf



(e) Blocked NW Australian Shelf

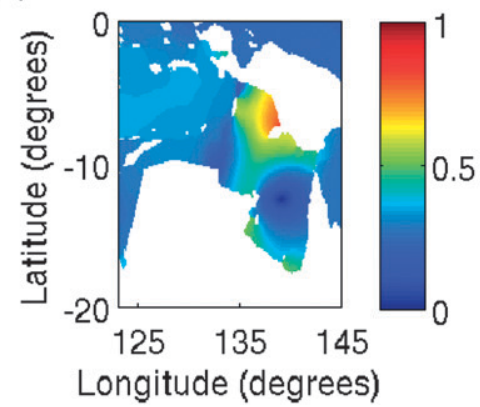

(c) Blocked Sahul Shelf - Unblocked

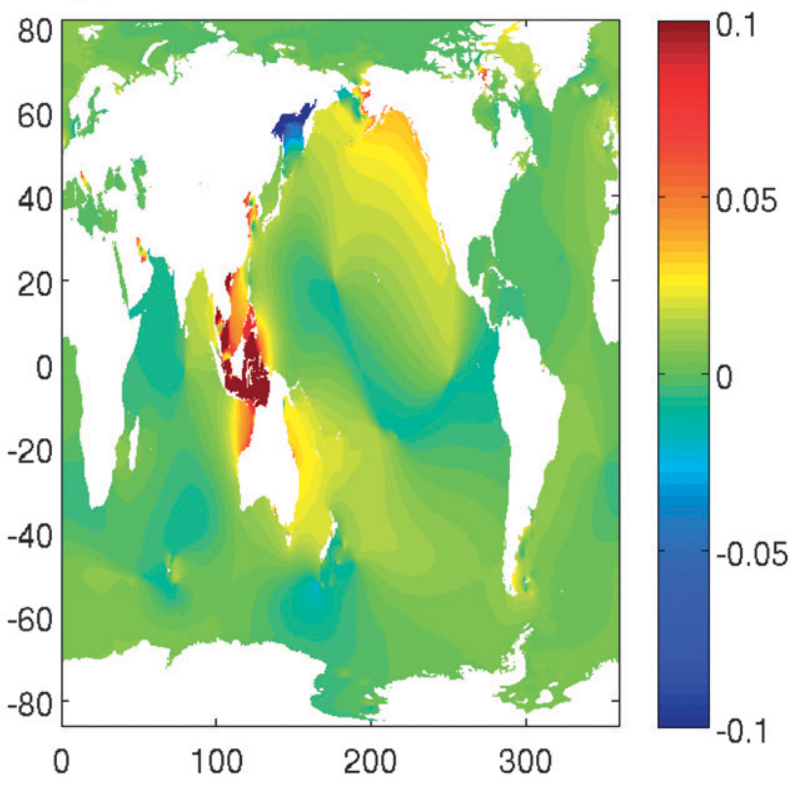

(f) Blocked NW Australian Shelf - Unblocked

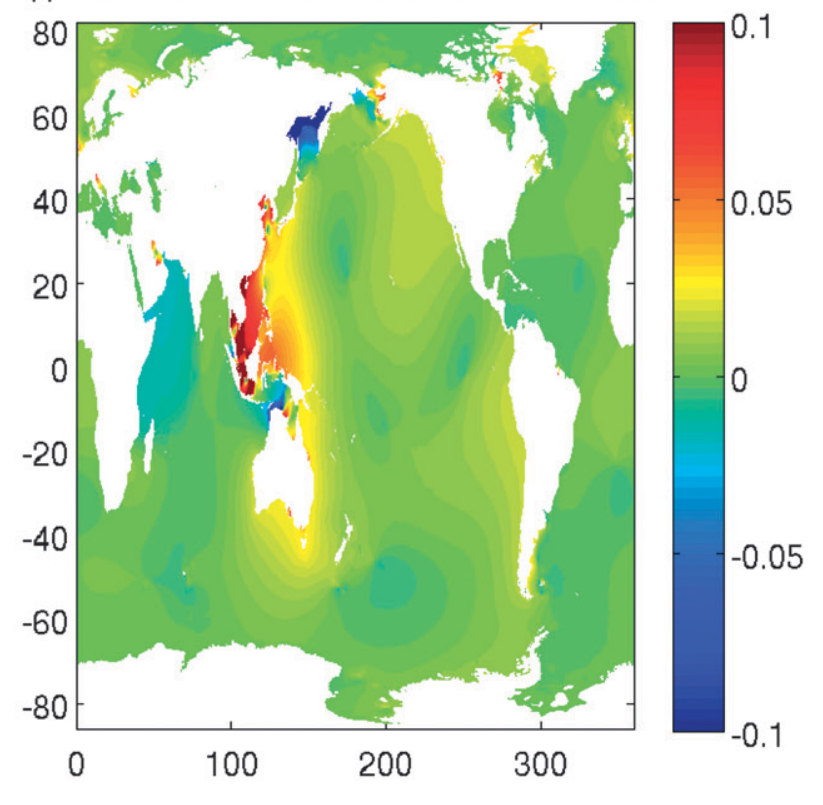

FIG. 12. As in Fig. 10, but for Blocked Sahul Shelf and Blocked northwest Australian Shelf. For the difference maps the scale is reduced from that in Fig. 10.

experiments described in this section, we also reran the $M_{2}$ Blocked Hudson Strait experiment in AKG at $1 / 8^{\circ}$ resolution and confirmed that it yielded similar results as the AKG Blocked Hudson Strait experiment, which was performed at $1 / 2$ resolution. The latter point demonstrates that the main AKG results are not artifacts of the relatively coarse horizontal resolution employed in that study.

\section{Summary and discussion}

We have systematically investigated the resonance of diurnal tides using a forward near-global ocean tide model and a modal synthesis (global free oscillation) model. The forward ocean tide simulations presented here build upon similar simulations conducted for 
(a) Unblocked Ross Sea



(b) Blocked Ross Sea

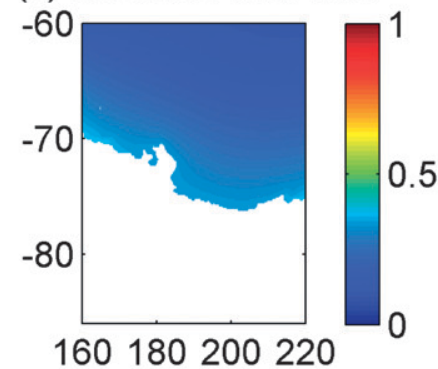

(d) Unblocked Gulf of Tonkin

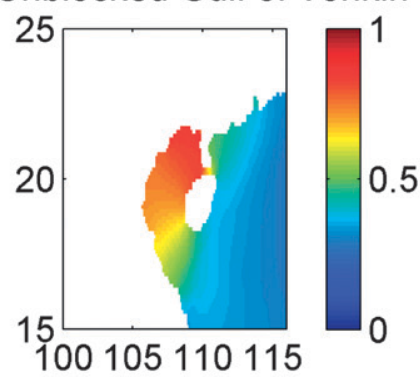

(e) Blocked Gulf of Tonkin



(c) Blocked Ross Sea - Unblocked



(f) Blocked Gulf of Tonkin - Unblocked

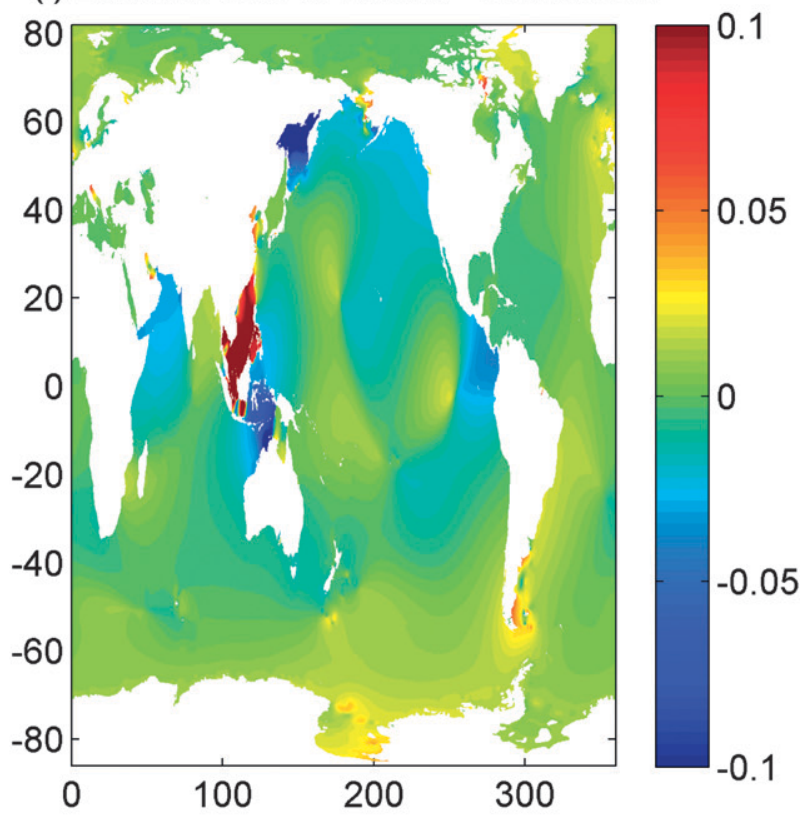

FIG. 13. As in Fig. 10, but for Blocked Ross Sea and Blocked Gulf of Tonkin. For the difference maps the scale is reduced from that in Fig. 10.

semidiurnal tides by AKG and AG. The set of global free oscillations (normal modes) computed for realistic ocean geometries and ocean physics are taken from Müller (2007, 2008, 2009).
Prior to conducting simulations with the forward ocean tide model, the topographic internal wave drag is tuned specifically for diurnal tides. In global tide models which are optimally tuned for $M_{2}$, kinetic energies for 
(a) Unblocked WCSA



(b) Blocked WCSA

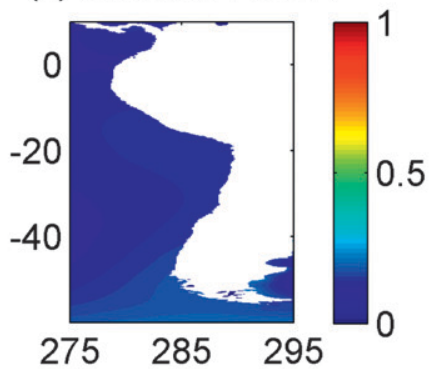

(d) Unblocked Bay of Bengal



(e) Blocked Bay of Bengal

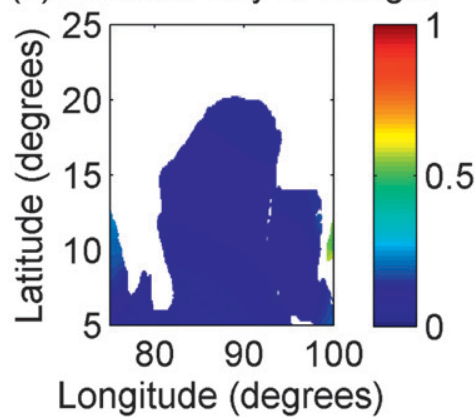

(c) Blocked WCSA - Unblocked



(f) Blocked Bay of Bengal - Unblocked

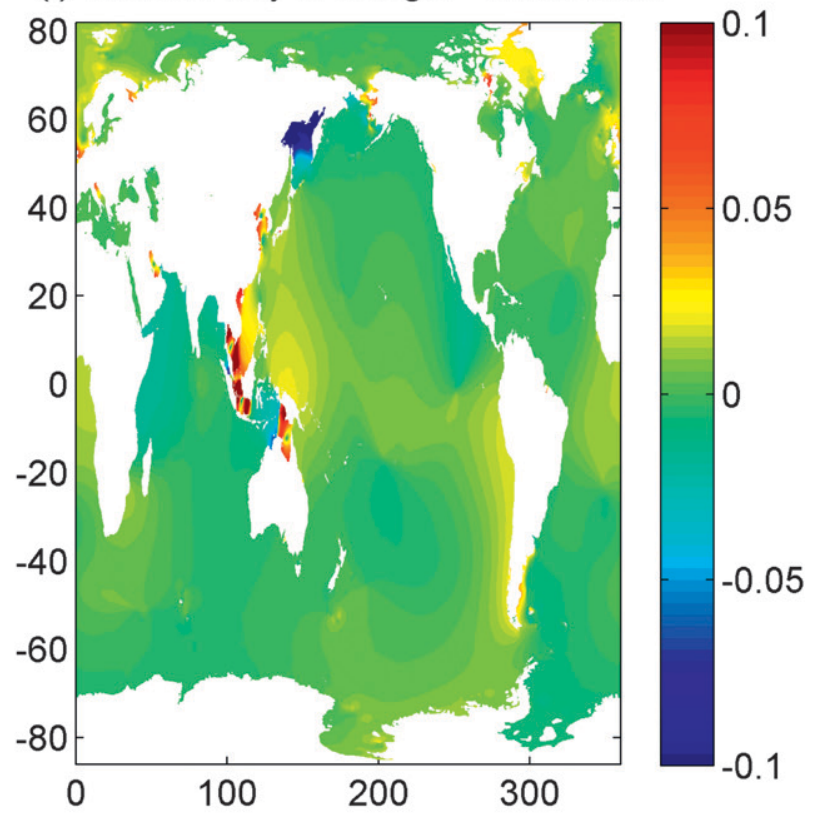

FIG. 14. As in Fig. 10, but for Blocked West Coast of South America and Blocked Bay of Bengal. For the difference maps the scale is reduced from that of Fig. 10.

the $K_{1}$ constituent are lower than those seen in current meter observations (P. Timko 2011, personal communication; Timko et al. 2012). Here, we adjust the wave drag by a well-known frequency-dependent factor and find that the discrepancies between the forward ocean tide model diurnal kinetic energies and those in satellitealtimetry-constrained tide models are substantially reduced as a result. 
(a) Unblocked Bass Strait

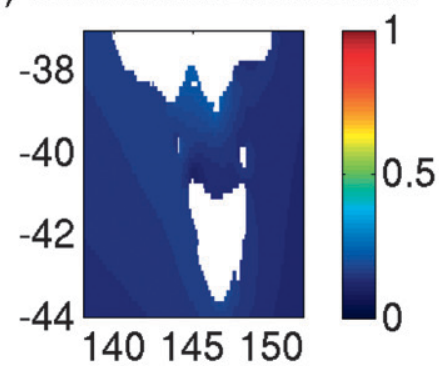

(b) Blocked Bass Strait

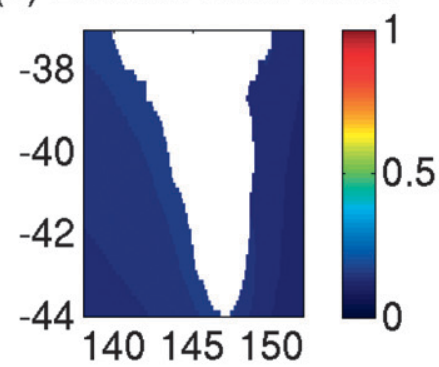

(d) Unblocked Gulf of Mexico

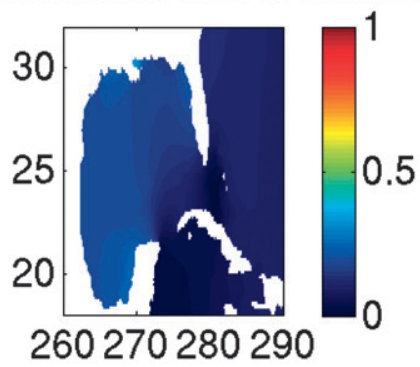

(e) Blocked Gulf of Mexico

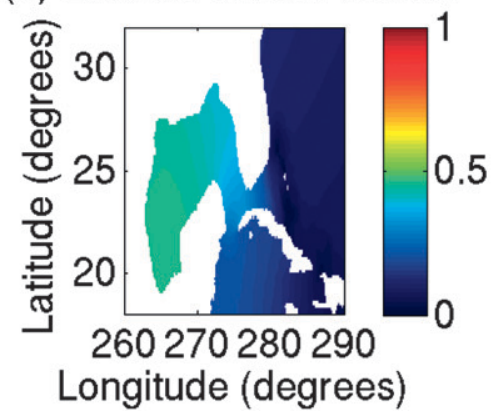

(c) Blocked Bass Strait - Unblocked

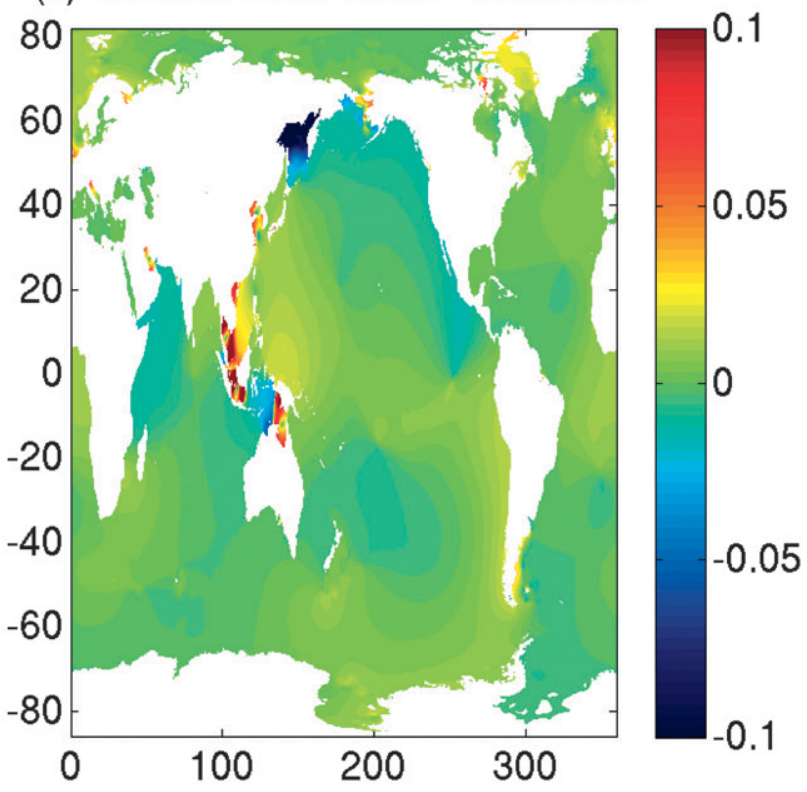

(f) Blocked Gulf of Mexico - Unblocked



FIG. 15. As in Fig. 10, but for Blocked Bass Strait and Blocked Gulf of Mexico. For the difference maps the scale is reduced from that in Fig. 10.

Simulations conducted with the forward ocean diurnal tide model and an array of forcing frequencies exhibit peaks in the globally averaged amplitude near periods of 22 and $33 \mathrm{~h}$. Predictions of sensitivity to forcing frequency made by the modal synthesis model closely mimic the results from the forward ocean tide simulations.
Specifically, the free oscillations with periods of 21.96 and $32.64 \mathrm{~h}$ yield peaks quantitatively consistent with the peaks near 22 and $33 \mathrm{~h}$ in the forward model results.

Sensitivity to zonal and meridional structure in the astronomical diurnal tidal forcing is also examined here. Forward ocean diurnal tide model simulations 
TABLE 4. Globally averaged diagnostics (see text) for various blocking experiments described in section 4e and shown in Figs. 10-15.

\begin{tabular}{lccccc}
\hline \multicolumn{1}{c}{ Region blocked out } & $\eta_{\mathrm{rms}}(\mathrm{cm})$ & $P(\mathrm{~cm})$ & Dissipation $(\mathrm{TW})$ & Area $\left(10^{11} \mathrm{~m}^{2}\right)$ & $P / \mathrm{area}^{2}\left[\mathrm{~cm}^{\left.\left(10^{11} \mathrm{~m}^{2}\right)^{-1}\right]}\right.$ \\
\hline Nominal (Unblocked-Fig. 1b) & 9.51 & - & 0.313 & - & - \\
Large Sea of Okhotsk & 10.1 & 3.00 & 0.302 & 14.8 & 0.20 \\
Small Sea of Okhotsk & 9.75 & 1.19 & 0.295 & 0.913 & 1.30 \\
Bristol Bay & 9.61 & 0.93 & 0.307 & 6.67 & 0.14 \\
Bering Sea & 9.63 & 1.25 & 0.312 & 12.9 & 0.18 \\
Sahul Shelf & 10.1 & 1.53 & 0.329 & 3.46 & 0.12 \\
Northwest Australian Shelf & 9.93 & 1.11 & 0.317 & 10.2 & 0.32 \\
Ross Sea & 9.64 & 1.25 & 0.290 & 3.73 & 0.12 \\
Gulf of Tonkin & 9.23 & 1.27 & 0.290 & 4.35 & 0.24 \\
West Coast South America & 9.44 & 0.73 & 0.298 & 1.16 & 0.20 \\
Bay of Bengal & 9.52 & 0.75 & 0.302 & 0.17 \\
Bass Strait & 9.48 & 0.68 & 0.300 & 0.298 & 0.59 \\
Gulf of Mexico & 9.29 & 2.20 & 0.29 & 0.29 \\
\hline
\end{tabular}

performed with variations in the zonal forcing structure show that globally averaged tidal amplitudes in the shelf regions peak at a zonal wavenumber equal to one. A broad peak near this wavenumber is also seen in the globally averaged open-ocean tidal amplitudes. The predictions made by the modal synthesis model once again resemble the forward ocean tide model results reasonably well. Forward ocean tide model simulations in which the meridional forcing structure is varied show that tidal elevations peak when the astronomical diurnal tidal forcing is of third degree. This result is consistent with predictions made from the modal synthesis model and with the relatively strong third-degree diurnal tides observed in the North Atlantic Ocean (Cartwright 1975; Ray 2001).

Taken together, the results presented here and in our previous studies of semidiurnal tides (AKG and AG) add to previously accumulated evidence (e.g., Platzman 1991; Müller 2008) that it is appropriate to view the semidiurnal and diurnal ocean tides as a system of forceddamped normal modes (free oscillations).

This paper also presents a discussion of diurnal tides in specific coastal locations and the impact of those locations on the open-ocean diurnal tides. Admittance analysis of the frequency sweep results demonstrates that the Sea of Okhotsk, Sahul Shelf, and Gulf of Tonkin are resonant to diurnal tidal forcing. Similarly, admittance analysis of the frequency sweep in AKG demonstrates that Hudson Strait is resonant to semidiurnal tidal forcing. A set of simulations in which specific coastal locations are blocked out demonstrates that blocking yields considerable alterations in tidal elevation amplitudes and phases, across basinwide and even global scales. Consistent with the predictions of simple analytical models in AG and AKG on the importance of coastal resonance, the Blocked Sea of Okhotsk and Blocked Hudson Strait experiments yield the largest perturbations to open-ocean diurnal and semidiurnal tides, respectively, of our blocked experiments. Regions of high diurnal tidal dissipation, such as the Bering Sea, yield large perturbations to the open-ocean diurnal tides as well, also in line with predictions from the simple analytical models in AG and AKG. Complicating the analysis, however, is the fact that blocking out regions in which neither diurnal tidal elevations nor dissipation are high, also often leads to significant perturbations to open-ocean diurnal tides. Evidently, the tides are quite sensitive to nearly any perturbations to geometry or to dissipation, particularly so in regions where the coastal tides are resonant. The blocking experiments are relevant to understanding tides of the ice age, during which lower sea levels entail a reduced area of continental shelves. Simulations of the ice age tides (e.g., Thomas and Sündermann 1999; Egbert et al. 2004; Arbic et al. 2004b, 2008; Uehara et al. 2006; Griffiths and Peltier 2008, 2009; Green 2010; Hill et al. 2011) strongly suggest that ice age tides were larger than those of the present day. The work presented in AKG, AG, and this paper provides some context with which to understand the results of ice-age tide simulations.

Acknowledgments. The authors thank two anonymous reviewers whose careful and extensive comments led to several important changes to this manuscript. BKA gratefully acknowledges support from National Science Foundation (NSF) Grants OCE-0924481 and OCE-09607820. AWS and LZ were supported by a Research Experience for Undergraduates (REU) supplement to OCE-09607820, while WJG was supported by an REU supplement to OCE-0924481. M.M. acknowledges support from DFG (Deutsche Forschungsgemeinschaft) Grant MU3009/1-1. We gratefully acknowledge Mike Messina for help in setting up the forward ocean tide model simulations for this project, which were carried 
out on the supercomputer provided by the University of Michigan Office of Research Cyberinfrastructure (ORCI). Important preliminary computations were done on the high-performance supercomputing cluster (HPC) at Florida State University. For the Florida State calculations BKA and WJG gratefully acknowledge assistance from Brian Rivera, Jordan Yao, Dan Voss, and Paul van der Mark.

\section{REFERENCES}

Arbic, B. K., and C. Garrett, 2010: A coupled oscillator model of shelf and ocean tides. Cont. Shelf Res., 30, 564-574, doi:10.1016/j.csr.2009.07.008.

__ S. T. Garner, R. W. Hallberg, and H. L. Simmons, 2004a: The accuracy of surface elevations in forward global barotropic and baroclinic tide models. Deep-Sea Res. II, 51, 3069-3101.

_ , D. R. MacAyeal, J. X. Mitrovica, and G. A. Milne, 2004b: Ocean tides and Heinrich events. Nature, 432, 460.

— , P. St-Laurent, G. Sutherland, and C. Garrett, 2007: On the resonance and influence of the tides in Ungava Bay and Hudson Strait. Geophys. Res. Lett., 34, L17606, doi:10.1029/ 2007GL030845.

—, J. X. Mitrovica, D. R. MacAyeal, and G. A. Milne, 2008: On the factors behind large Labrador Sea tides during the last glacial cycle and the potential implications for Heinrich events. Paleoceanography, 23, PA3211, doi:10.1029/2007PA001573.

__ R. H. Karsten, and C. Garrett, 2009: On tidal resonance in the global ocean and the back-effect of coastal tides upon open-ocean tides. Atmos.-Ocean, 47, 239-266, doi:10.3137/ OC311.2009.

Arfken, G. B., and H. J. Weber, 2001: Mathematical Methods For Physicists. 5th ed. Academic Press, 1096 pp.

Carrere, L., and F. Lyard, 2003: Modeling the barotropic response of the global ocean to atmospheric wind and pressure forcingcomparisons with observations. Geophys. Res. Lett., 30, 1275, doi:10.1029/2002GL016473.

Cartwright, D. E., 1975: A subharmonic lunar tide in the seas off Western Europe. Nature, 257, 277-280.

_ 1977: Ocean tides. Rep. Prog. Phys., 40, 665-708.

Clarke, A. J., 1991: The dynamics of barotropic tides over the continental shelf and slope (review). Tidal Hydrodynamics, B. B. Parker, Ed., John Wiley and Sons, 79-108.

Cummins, P. F., R. H. Karsten, and B. K. Arbic, 2010: The semidiurnal tide in Hudson Strait as a resonant channel oscillation. Atmos.-Ocean, 48, 163-176, doi:10.3137/OC307.2010.

Defant, A., 1961: Physical Oceanography. Vol. 2. Pergamon Press, $598 \mathrm{pp}$.

Egbert, G. D., and R. D. Ray, 2000: Significant dissipation of tidal energy in the deep ocean inferred from satellite altimeter data. Nature, 405, 775-778.

- , and — 2001: Estimates of $\mathrm{M}_{2}$ tidal energy dissipation from TOPEX/Poseidon altimeter data. J. Geophys. Res., 106 (C10), 22 475-22 502.

— and - 2003: Semi-diurnal and diurnal tidal dissipation from TOPEX/Poseidon altimetry. Geophys. Res. Lett., 30, 1907, doi:10.1029/2003GL017676.

—_ A. F. Bennett, and M. G. G. Foreman, 1994: TOPEX/ Poseidon tides estimated using a global inverse model. J. Geophys. Res., 99 (C12), 24 821-24 852.
- R. D. Ray, and B. G. Bills, 2004: Numerical modeling of the global semidiurnal tide in the present day and in the last glacial maximum. J. Geophys. Res., 109, C03003, doi:10.1029/ 2003 JC001973.

Garner, S. T., 2005: A topographic drag closure built on an analytical base flux. J. Atmos. Sci., 62, 2302-2315.

Garrett, C., 1972: Tidal resonance in the Bay of Fundy and Gulf of Maine. Nature, 238, 441-443.

— The open boundary problem. J. Phys. Oceanogr., 7, 171-181.

Gill, A. E., 1982: Atmosphere-Ocean Dynamics. Academic Press, $662 \mathrm{pp}$.

Godin, G., 1988: Tides. Anadyomene, 348 pp.

Green, J. A. M., 2010: Ocean tides and resonance. Ocean Dyn., 60, 1243-1253, doi:10.1007/s10236-010-0331-1.

Griffiths, S. D., and W. R. Peltier, 2008: Megatides in the Arctic Ocean under glacial conditions. Geophys. Res. Lett., 35, L08605, doi:10.1029/2008GL033263.

— , and — 2009: Modelling of polar ocean tides at the Last Glacial Maximum: Amplification, sensitivity, and climatological implications. J. Climate, 22, 2905-2924.

Heath, R. A., 1981: Estimates of the resonant period and Q in the semi-diurnal tidal band in the North Atlantic and Pacific Oceans. Deep-Sea Res., 28, 481-493.

Hendershott, M. C., 1972: The effects of solid earth deformation on global ocean tides. Geophys. J. Roy. Astron. Soc., 29, 389-402.

- 1981: Long waves and ocean tides. Evolution of Physical Oceanography: Scientific Surveys in Honor of Henry Stommel, B. A. Warren and C. Wunsch, Eds., The MIT Press, 291-341. Hill, D. F., S. D. Griffiths, W. R. Peltier, B. P. Horton, and T. E. Tornqvist, 2011: High-resolution numerical modeling of tides in the western Atlantic, Gulf of Mexico, and Caribbean Sea during the Holocene. J. Geophys. Res., 116, C10014, doi:10.1029/2010JC006896.

Jayne, S. R., and L. C. St. Laurent, 2001: Parameterizing tidal dissipation over rough topography. Geophys. Res. Lett., 28, 811-814.

Laplace, P. S., 1775: Recherches sur plusieurs points du système du monde. Mem. Acad. Roy. Sci. Paris, 88, 75-182. [Reprinted in Oeuvres Complètes de Laplace, Gauthier-Villars, Paris, 9 (1893)].

— 1776: Recherches sur plusieurs points du système du monde. Mem. Acad. Roy. Sci. Paris, 89, 177-264. [Reprinted in Oeuvres Complètes de Laplace, Gauthier-Villars, Paris, 9 (1893)].

Longuet-Higgins, M. S., and G. S. Pond, 1970: The free oscillations of fluid on a hemisphere bounded by meridians of longitude. Philos. Trans. Roy. Soc. London, A266, 193-223.

Lyard, F., F. Lefevre, T. Letellier, and O. Francis, 2006: Modelling the global ocean tides: Modern insights from FES2004. Ocean Dyn., 56, 394-415, doi:10.1007/s10236-006-0086-x.

Müller, M., 2007: The free oscillations of the world ocean in the period range 8 to 165 hours including the full loading effect. Geophys. Res. Lett., 34, L05606, doi:10.1029/2006GL028870.

_ 2008: Synthesis of forced oscillations, Part I: Tidal dynamics and the influence of the loading and self-attraction effect. Ocean Modell., 20, 207-222, doi:10.1016/j.ocemod.2007.09.001.

, 2009: A Large Spectrum of Free Oscillations of the World Ocean Including the Full Ocean Loading and Self-Attraction Effects. Vol. 14, Hamburg Studies on Maritime Affairs, 118 pp.

, B. K. Arbic, and J. X. Mitrovica, 2011: Secular trends in ocean tides: Observations and model results. J. Geophys. Res., 116, C05013, doi:10.1029/2010JC006387.

Platzman, G. W., 1984: Normal modes of the World Ocean. Part IV: Synthesis of diurnal and semidiurnal tides. J. Phys. Oceanogr., 14, 1532-1550. 
1991: Tidal evidence for ocean normal modes. Tidal Hydrodynamics, B. B. Parker, Ed., John Wiley and Sons, 13-26.

- , G. A. Curtis, K. S. Hansen, and R. D. Slater, 1981: Normal modes of the world ocean. Part II: Description of modes in the period range 8 to 80 hours. J. Phys. Oceanogr., 11, 579-603.

Polzin, K. L., J. M. Toole, J. R. Ledwell, and R. W. Schmitt, 1997: Spatial variability of turbulent mixing in the abyssal ocean. Science, 276, 93-96.

Proudman, J., 1953: Dynamical Oceanography. Methven and Co., 409 pp.

Rao, D. B., 1966: Free gravitational oscillations in rotating rectangular basins. J. Fluid Mech., 25, 523-555.

Ray, R. D., 1999: A global ocean tide model from TOPEX/ POSEIDON altimetry: GOT99.2. National Aeronautics and Space Administration Tech. Memo. NASA/TM-1999209478, 58 pp.

2001: Resonant third-degree diurnal tides in the seas off Western Europe. J. Phys. Oceanogr., 31, 3581-3586.

Simmons, H. L., R. W. Hallberg, and B. K. Arbic, 2004: Internal wave generation in a global baroclinic tide model. Deep-Sea Res., 51, 3043-3068.
Sutherland, G., C. Garrett, and M. Foreman, 2005: Tidal resonance in Juan de Fuca Strait and the Strait of Georgia. J. Phys. Oceanogr., 35, 1279-1286.

Thomas, M., and J. Sündermann, 1999: Tides and tidal torques of the world ocean since the last glacial maximum. J. Geophys. Res., 104 (C2), 3159-3183.

Timko, P. G., B. K. Arbic, J. G. Richman, R. B. Scott, E. J. Metzger, and A. J. Wallcraft, 2012: Skill tests of threedimensional tidal currents in a global ocean model: A look at the North Atlantic. J. Geophys. Res., 117, C08014, doi:10.1029/2011JC007617.

Uehara, K., J. D. Scourse, K. J. Horsburgh, K. Lambeck, and A. P. Purcell, 2006: Tidal evolution of the northwest European shelf seas from the Last Glacial Maximum to the present. J. Geophys. Res., 111, C09025, doi:10.1029/2006JC003531.

Wunsch, C., 1972: Bermuda sea level in relation to tides, weather, and baroclinic fluctuations. Rev. Geophys., 10, 1-49.

Zahel, W., and M. Müller, 2005: The computation of the free barotropic oscillations of a global ocean model including friction and loading effects. Ocean Dyn., 55, 137-161, doi:10.1007/ s10236-005-0029-y. 